\title{
GENERALIZED CLASS OF MEAN ESTIMATORS FOR TWO PHASE SAMPLING IN THE PRESENCE OF NONRESPONSE
}

\author{
Zahoor Ahmad*, IrsaZafar* and Zakia Bano*
}

\begin{abstract}
A lot of work has been done in two-phase sampling for estimating the population mean of a study variable while considering the non-response at the second phase, see e.g. Khare and Srivastava (1993, 1995), Tabasum and Khan (2004), Singh and Kumar (2008) and Singh et al. (2010). Most authors have used information of a single auxiliary variable while estimating the mean of the study variable, whereas in a practical situation we may require/have auxiliary information on multiple characters. Khare and Sinha $(2009,2011)$ have used multi-auxiliary characters to estimate the population mean in the presence of nonresponse in the case of simple random sampling. In two-phase sampling, when auxiliary information is obtained at both phases, the nonresponse may occur at both phases as well. In this paper we have proposed a generalized class of estimators for estimating the population mean of a study variable under a two-phase sampling scheme using multi-auxiliary variable(s) in the presence of nonresponse at both phases. The information on all auxiliary variable(s) is not known for the population. The bias and mean square error have been derived for the suggested class. Special cases of the class have also been identified. An empirical study has been conducted for comparing the efficiency of the proposed estimators with a modified version of existing ones.
\end{abstract}

Key words and phrases: Generalized class of estimators, multi-auxiliary variables, non-response, two phase sampling.

\section{Introduction}

The use of auxiliary information can increase the precision of an estimator when study variable $y$ is highly correlated with the auxiliary variable $x$ (see Singh et al. (2010)). In single phase sampling, while estimating the population mean by ratio, regression etc. methods of estimation, the auxiliary information in the form of a population parameter is required; when such information is not available, a two-phase sampling scheme can be used where a large first-phase sample measured over the auxiliary variable $x$ is used to obtain a good estimate of population parameters. A second-phase sample can then be taken and the study variable $y$ with an auxiliary variable $x$ can be observed. Wu and Luan (2003) discussed that the major advantage of using two-phase sampling is the gain in high precision without a substantial increase in cost.

The presence or absence of population auxiliary information plays an important role in the efficiency of estimators for two-phase sampling. Samiuddin and Hanif (2007) first discussed that there are three possible cases for the use of auxiliary information; no information case (when population information about none

\footnotetext{
Received July 2, 2013. Revised November 6, 2013. Accepted December 2, 2013.

*University of Gujrat, Gujrat, Pakistan. Email: zahoor.ahmed@uog.edu.pk
} 
of the auxiliary variables is available), partial information case (when population information on some auxiliary variables is available) and full information case (when population information on all auxiliary variables is available). Ahmad and Hanif (2010) emphasize that the estimators developed by using the same technique, for example regression or ratio, should be compared on the basis of the mean square error within these three cases because it is obvious that an estimator will be more efficient for the full information case as compared to partial and no. The same is true for partial and no information cases. The trend of using multi-auxiliary variables is increasing due to practical requirements. The above three cases can be considered for the use of multi-auxiliary information and in this manner the precision of the estimates can also be improved. Recently under these three cases, several classes of univariate and multivariate ratio and regression estimators have been developed in two-phase sampling by Ahmad et al. (2009a, 2009b, 2010) and Ahmad and Hanif (2010).

The most common method of data collection in survey research is sending questionnaires through the mail. The reason may be due to the minimum cost involved in this method. But this method has a major disadvantage in that, a high rate of non-response may occur. This may result in an unknown bias at any assumption because of the fact that the estimate based only on responding units is representative of both the responding and non-responding units. Personal interview is another method of data collection which generally may result in a complete response, but the cost involved in personal interviews is much higher than the mail questionnaire method. We may conclude from the above discussion that the advantage of one method is the disadvantage of the other and vice versa. Hansen and Hurwitz (1946) combined the advantages of both procedures. They considered a problem to determine the number of mail questionnaires along with the number of personal interviews to conduct in following up to non-response to the mail questionnaire, in order to attain the required precision at minimum cost.

Considering the above approach, let us assume that the population of size $N$ is divided into two groups, one group consisting of the units who respond, called the response class, and the other group consisting of the units who do not respond, called the non-response class. Let $N_{1}$ and $N_{2}$ be the sizes of the responding class (denoted by $U_{1}$ ) and the non-responding class (denoted by $U_{2}$ ) of the population respectively. A sample of size $n_{1}$ (denoted by $u_{1}$ ) is selected from the population of $N$ units and it is assumed that $n_{11}$ units (denoted by $v_{1}^{\prime}$ ) respond and $n_{12}$ (denoted by $v_{2}^{\prime}$ ) do not, where $v_{1}^{\prime}=u_{1} \cap U_{1}$ and $v_{2}^{\prime}=u_{1} \cap U_{2}$. A sub-sample (denoted by $v_{2 m}^{\prime}$ ) of $r_{1}$ units out of $n_{12}$ is re-contacted such that $r_{1}=\frac{n_{12}}{k_{1}}, k_{1}>1$. Under this sampling scheme, Hansen and Hurwitz (1946) constructed the following unbiased estimator for population mean $\bar{Y}$ of variable of interest $y$ as

$$
\bar{y}_{1}^{*}=w_{1} \bar{y}_{1}+w_{1}^{\prime} \bar{y}_{1 r},
$$

where $\bar{y}_{1}$ and $\bar{y}_{1 r}$ denote the means of the study variable, for respondent and recontacted samples respectively, and the weights $w_{1}=n_{11} / n_{1}$ and $w_{1}^{\prime}=n_{12} / n_{1}$. 
The variance of (1.1) is

$$
\operatorname{Var}\left(\bar{y}_{1}^{*}\right)=\left(N-n_{1}\right)\left(n_{1} N\right)^{-1} S_{y}^{2}+\frac{W_{2}\left(k_{1}-1\right)}{n_{1}} S_{y_{2}}^{2},
$$

where $S_{y}^{2}=(N-1)^{-1} \sum_{j=1}^{N}\left(y_{j}-\bar{Y}\right)^{2}$ and $S_{y_{2}}^{2}=\left(N_{2}-1\right) \sum_{j=1}^{N_{2}}\left(y_{j}-\bar{Y}_{2}\right)^{2}$ are population variances for responding and nonresponding portions with means $\bar{Y}=$ $(N)^{-1} \sum_{i=1}^{N} y_{i}$ and $\bar{Y}_{2}=\left(N_{2}\right)^{-1} \sum_{i=1}^{N_{2}} y_{i}, W_{2}=N_{2}(N)^{-1}$.

In the case of nonresponse for simple random sampling, Tripathi and Khare (1997) considered the estimation of mean vector for partial and complete nonresponse. Khare and Sinha (2009) proposed two classes of estimators of mean in the presence of non-response using multi-auxiliary variables. Further, Khare and Sinha (2011) considered class of estimators for population mean using multiauxiliary characters with unknown means in the presence of non-response.

After introducing the concept of two-phase sampling, use of auxiliary information and non-response in Section 1, Section 2 contains the two-phase sampling scheme with important developments in estimating population mean using single auxiliary information in the presence of non-response in the second phase. For the multi-auxiliary information case, the same sampling scheme, some important notations and results are discussed in Section 3. In Section 4, we have proposed a very general class of regression-cum-ratio-exponential estimator for estimating the mean of a study variable in the presence of non-response at both phases, and its special cases are also given in this section. Detailed empirical study has been conducted and discussed in Section 5. Finally some conclusions are provided.

\section{Two-phase sampling in the presence of non-response in the second phase using a single auxiliary variable}

Consider the population of $N$ units, and further it is assumed that from $N$, $N_{1}$ units (denoted by $U_{1}$ ) belong to the respondent group and $N_{2}$ units (denoted by $U_{2}$ ) belong to non-respondents. From $N$ units, a first phase sample (denoted by $u_{1}$ ) of $n_{1}$ units is drawn by simple random sampling without replacement (SRSWOR). A second phase sample (denoted by $u_{2}$ ) of $n_{2}$ units (i.e. $n_{2}<n_{1}$ ) is drawn from $n_{1}$ by simple random sampling without replacement (SRSWOR) and the variable of interest $y$ is measured on it. In the second phase let $n_{21}$ units supply information which is denoted by $v_{1}$ and $n_{22}$ units refuse to respond which is denoted by $v_{2}$, where $v_{1}=u_{2} \cap U_{1}$ and $v_{2}=u_{2} \cap U_{2}$. A sub-sample (denoted by $v_{2 m}$ ) of $r_{2}$ units is randomly taken from the $n_{22}$ non-respondents by applying the method of Hansen and Hurwitz (1946) and this sub sample is specified by $r_{2}=n_{22} / k_{2}, k_{2}>1$. Assume that no non response is observed in this sub sample. Following Hansan and Hurwitz (1946), let $\bar{y}_{2}^{*}$ and $\bar{x}_{2}^{*}$ denote the estimate of population mean $\bar{Y}$ and $\bar{X}$ respectively in the second phase with nonresponse and let $\bar{x}_{1}$ denote the sample mean of the auxiliary variable in the first phase. Under this sampling scheme the following are the wellknown estimators for estimating the population mean of a study variable using auxiliary information in the presence of non-response. 
The simple random sampling version of mean estimator in the presence of non-response suggested by Hansen and Hurwitz (1946) may be modified in the case of two-phase sampling when non-response is considered at the second phase as:

$$
\bar{y}_{2}^{*}=w_{2} \bar{y}_{2}+w_{2}^{\prime} \bar{y}_{2 r},
$$

where $\bar{y}_{2}$ and $\bar{y}_{2 r}$ denote the means of study variables for respondent and recontacted samples in the second phase, and $w_{2}=n_{21} / n_{2}$ and $w_{2}^{\prime}=n_{22} / n_{2}$ where $n_{2}$ and $n_{22}$ are respondent and non-respondent respectively, sample sizes in the second phase. The expression of variance is

$$
\operatorname{Var}\left(\bar{y}_{2}^{*}\right)=\left(N-n_{2}\right)\left(n_{2} N\right)^{-1} S_{y}^{2}+\frac{W_{2}\left(k_{2}-1\right)}{n_{2}} S_{y_{2}}^{2} .
$$

Khare and Srivastava $(1993,1995)$ proposed ratio, product and regression estimators in the presence of non-response at the second phase. Their ratio estimator is

$$
\begin{aligned}
& T_{R 1 d}=\frac{\bar{y}_{2}^{*}}{\bar{x}_{2}^{*}} \bar{x}_{1} \\
& \begin{aligned}
\operatorname{Bias}\left(T_{R 1 d}\right) \approx\left(\bar{X}^{-1}\right)\left[\left(n_{2}\right)^{-1}-\left(n_{1}\right)^{-1}\left(R S_{x}^{2}-S_{x y}\right)\right. \\
\left.\quad+W_{2}\left(k_{2}-1\right)\left(n_{2}\right)^{-1}\left(R S_{x_{2}}^{2}-S_{x y_{(2)}}\right)\right]
\end{aligned}
\end{aligned}
$$

and

$$
\begin{aligned}
\operatorname{MSE}\left(T_{R 1 d}\right) \approx & \left(N-n_{1}\right)\left(n_{1} N\right)^{-1} S_{y}^{2}+\left(n_{1}-n_{2}\right)\left(n_{1} n_{2}\right)^{-1} S_{r}^{2} \\
& +W_{2}\left(k_{2}-1\right)\left(n_{2}\right)^{-1} S_{r_{2}}^{2},
\end{aligned}
$$

where $S_{r}^{2}=\left\{S_{y}^{2}+R^{2} S_{x}^{2}(1-2 C)\right\}, S_{r_{2}}^{2}=\left\{S_{y_{2}}^{2}+R^{2} S_{x_{2}}^{2}\left(1-2 C_{2}\right)\right\}, C=\frac{\beta}{R}$ and $C_{(2)}=\frac{\beta_{(2)}}{R}$.

The product estimator is

$$
\begin{aligned}
& T_{P 1 d}=\frac{\bar{y}_{2}^{*}}{\bar{x}_{1}} \bar{x}_{2}^{*}, \\
& \operatorname{Bias}\left(T_{P 1 d}\right) \approx \frac{1}{\bar{X}}\left[\left(n_{1}-n_{2}\right)\left(n_{1} n_{2}\right)^{-1} S_{x y}+W_{2}\left(k_{2}-1\right)\left(n_{2}\right)^{-1} S_{x y_{(2)}}\right],
\end{aligned}
$$

and

$$
\begin{aligned}
\operatorname{MSE}\left(T_{P 1 d}\right) \approx & \left(N-n_{1}\right)\left(n_{1} N\right)^{-1} S_{y}^{2}+\left(n_{1}-n_{2}\right)\left(n_{1} n_{2}\right)^{-1} S_{p}^{2} \\
& +W_{2}\left(k_{2}-1\right)\left(n_{2}\right)^{-1} S_{p_{2}}^{2},
\end{aligned}
$$

where $S_{p}^{2}=\left\{S_{y}^{2}+R^{2} S_{x}^{2}(1+2 C)\right\}, S_{p_{2}}^{2}=\left\{S_{y_{2}}^{2}+R^{2} S_{x_{2}}^{2}\left(1+2 C_{2}\right)\right\}$.

The regression estimator is

$$
T_{L R 1 d}=\bar{y}_{2}^{*}+b^{*}\left(\bar{x}_{1}-\bar{x}_{2}^{*}\right), \quad \text { where } \quad b^{*}=\frac{S_{x y}}{S_{x}^{2}},
$$




$$
\begin{aligned}
\operatorname{Bias}\left(T_{L R 1 d}\right) \approx \beta_{x y} & \frac{N\left(N-n_{1}\right)}{(N-1)(N-2)} \frac{\mu_{21}}{n_{1} \bar{X} S_{y x}} \\
& -\frac{N\left(N-n_{1}\right)}{(N-1)(N-2)} \frac{\mu_{30}}{n_{1} \bar{X} S_{x}^{2}}+\theta_{2} \frac{\mu_{30_{(2)}}}{\bar{X} S_{x}^{2}} \\
& +\frac{N\left(N-n_{2}\right)}{(N-1)(N-2)} \frac{\mu_{30}}{n_{2} \bar{X} S_{x}^{2}} \\
& \left.-\frac{N\left(N-n_{2}\right)}{(N-1)(N-2)} \frac{\mu_{21}}{n_{2} \bar{X} S_{y x}}-\theta_{2} \frac{\mu_{21_{(2)}}}{\bar{X} S_{y x}}\right)
\end{aligned}
$$

where

$$
\mu_{v s}=\frac{1}{N-1} \sum_{i=1}^{N}\left(x_{i}-\bar{X}\right)^{v}\left(y_{i}-\bar{Y}\right)^{s}
$$

and

$$
\mu_{v s_{(2)}}=\frac{1}{\left(N_{2}-1\right)} \sum_{i=1}^{N_{2}}\left(x_{i}-\bar{X}_{2}\right)^{v}\left(y_{i}-\bar{Y}_{2}\right)^{s},
$$

$(v, s)$ being nonnegative integers.

Also

$$
\begin{aligned}
\operatorname{MSE}\left(T_{L R 1 d}\right)= & \left(N-n_{1}\right)\left(n_{1} N\right)^{-1} S_{y}^{2}+\left(n_{1}-n_{2}\right)\left(n_{1} n_{2}\right)^{-1} S_{y}^{2}\left(1-\rho^{2}\right) \\
& +W_{2}\left(k_{2}-1\right)\left(n_{2}\right)^{-1} S_{\beta_{2}}^{2},
\end{aligned}
$$

where $S_{\beta_{2}}^{2}=\left[S_{y_{2}}^{2}+\beta S_{x_{2}}^{2}\left(\beta-2 \beta_{(2)}\right)\right]$.

Tabasum and Khan (2004) revisited the Khare and Srivastava (1993) ratio estimator $T_{R 1 d}$ and found that the cost of this estimator is less than the cost calculated by Hansen-Hurwitz (1946) estimators under certain conditions. Singh et al. (2010) suggested the following generalized version of modified exponential ratio and exponential product type estimators using two-phase sampling.

$$
t_{(a)}^{(1)}=\bar{y}_{2}^{*} \exp \left[\frac{a\left(\bar{x}_{2}^{*}-\bar{x}_{1}\right)}{\left(\bar{x}_{2}^{*}+\bar{x}_{1}\right)}\right],
$$

with

$$
\begin{aligned}
\operatorname{Bias}\left(t_{(a)}^{(1)}\right)=\frac{a}{2 \bar{X}}[ & \left(n_{1}-n_{2}\right)\left(n_{1} n_{2}\right)^{-1}\left(S_{x y}-\frac{R}{2} S_{x}^{2}\right) \\
& \left.+W_{2}\left(k_{2}-1\right)\left(n_{2}\right)^{-1}\left(S_{x y_{(2)}}-\frac{R}{2} S_{x_{2}}^{2}\right)\right]
\end{aligned}
$$

and

$$
\operatorname{MSE}\left(t_{(a)}^{(1)}\right)=\left(n_{1}-n_{2}\right)\left(n_{1} n_{2}\right)^{-1}\left[S_{y}^{2}+\left(\frac{a R^{2} S_{x}^{2}}{4}\right)(1+4 C)\right]
$$




$$
\begin{aligned}
& +\left(N-n_{1}\right)\left(n_{1} N\right)^{-1} S_{y}^{2} \\
& +W_{2}\left(k_{2}-1\right)\left(n_{2}\right)^{-1}\left[S_{y_{2}}^{2}+\left(\frac{a R^{2} S_{x_{2}}^{2}}{4}\right)\left(1+4 C_{(2)}\right)\right] .
\end{aligned}
$$

When $a=-1$ and 1 , the expression given in (2.12) becomes an exponential-ratioestimator and exponential-product estimator along with their bias and mean square error respectively.

\section{Two-phase sampling using multi-auxiliary variables in the presence of non-response at both phases}

In two-phase sampling, when auxiliary information is obtained from the first phase sample that is relatively larger than the second phase, the non-response rate will be larger in the first phase sample. A lot of literature is devoted to the case when nonresponse occurs in the second phase. Hence there is a need to develop estimators when non-response is considered in both phases. In this paper, a class of generalized estimators in the presence of nonresponse in both phases is developed. It is also considered that information on all auxiliary variables is not known (No Information Case) that usually occurs in most practical situations.

After discussing the sampling scheme of Section 2, from the first phase sample (denoted by $u_{1}$ ) of $n_{1}$ units we assume that $n_{11}$ units supply information which is denoted by, and $n_{12}$ units refuse to respond which is denoted by $v_{2}^{\prime}$, where $v_{1}^{\prime}=u_{1} \cap U_{1}$ and $v_{2}^{\prime}=u_{1} \cap U_{2}$. A sub-sample (denoted by $v_{2 m}^{\prime}$ ) of $r_{1}$ units is randomly taken from the $n_{12}$ non-respondents by applying the method of Hansen and Hurwitz (1946) and this sub sample is specified by $r_{1}=n_{12} / k_{1}, k_{1}>1$.

In the Hansen and Hurwitz (1946) approach it is assumed that no non response is observed in the sub sample of non-respondents. But it is not a realistic assumption in recent sampling and it is possible that non-response may appear in re-contracted samples.

Let, $x_{i}$ denote the $i$ th auxiliary variable for all $i=1,2,3, \ldots, q$ with population mean $\bar{X}_{i}=N^{-1} \sum_{J=1}^{N} X_{i J}, \bar{X}_{1 i}=N_{1}^{-1} \sum_{J^{\prime}=1}^{N_{1}} X_{1 i J^{\prime}}$ and $\bar{X}_{2 i}=$ $N_{2}^{-1} \sum_{L=1}^{N_{2}} X_{2 i L}$ denote the population means of the $i$ th auxiliary variable for the response and non-response groups respectively, and $S_{x_{i}}^{2}=(N-1)^{-1} \sum_{J=1}^{N}\left(X_{i J}-\right.$ $\left.\bar{X}_{i}\right)^{2}$ and $S_{x_{2 i}}^{2}=\left(N_{2}-1\right)^{-1} \sum_{L=1}^{N_{2}}\left(X_{2 i L}-\bar{X}_{2 i}\right)^{2}$ denote the variances of the $i$ th auxiliary variable for the complete population and non-respondents respectively. $S_{x_{i} x_{i^{\prime}}}=(N-1)^{-1} \sum_{J=1}^{N}\left(X_{i J}-\bar{X}_{i}\right)\left(X_{i^{\prime} J}-\bar{X}_{i^{\prime}}\right)$ and $S_{x_{i} x_{i^{\prime}(2)}}=\left(N_{2}-\right.$ $1)^{-1} \sum_{L=1}^{N_{2}}\left(X_{i L}-\bar{X}_{2 i}\right)\left(X_{i^{\prime} L}-\bar{X}_{2 i^{\prime}}\right)$ denote the co-variances between the two auxiliary variables for complete population and the population of non-respondents respectively, and $S_{x_{i} y}=(N-1)^{-1} \sum_{J=1}^{N}\left(X_{i J}-\bar{X}_{i}\right)\left(y_{J}-\bar{Y}\right)$ and $S_{x_{i} y_{(2)}}=$ $\left(N_{2}-1\right)^{-1} \sum_{L=1}^{N_{2}}\left(x_{i L}-\bar{X}_{2 u}\right)\left(y_{i L}-\bar{Y}_{2}\right)$ denote the co-variances between the study and auxiliary variable for complete population and the population of nonrespondents respectively.

Let $\bar{x}_{1 i}=n_{11}^{-1} \sum_{j=1}^{n_{11}} x_{1 i j}$ and $\bar{x}_{2 i}=n_{21}^{-1} \sum_{l=1}^{n_{21}} x_{2 i l}$ denote the sample means of the $i$ th auxiliary variable for the first and second phase responding units respectively, and $\bar{x}_{r^{1} i}=\frac{1}{r_{1}} \sum_{j^{\prime}=1}^{r_{1}} x_{r^{1} i j^{\prime}}$ and $\bar{x}_{r^{2} i}=\frac{1}{r_{2}} \sum_{l^{\prime}=1}^{r_{2}} x_{r^{1} i l^{\prime}}$ denote the sample 
means of the $i$ th auxiliary variable for sub-sampled units in the first phase and second phase respectively.

Following Hansen and Hurwitz (1946) approach, $\bar{x}_{1 i}^{*}=n_{1}^{-1}\left(n_{11} \bar{x}_{1 i}+n_{12} \bar{x}_{r^{1} i}\right)$ and $\bar{x}_{2 i}^{*}=n_{2}^{-1}\left(n_{21} \bar{x}_{2 i}+n_{22} \bar{x}_{r^{2} i}\right)$ denote sample means of the $i$ th auxiliary variable for first phase and second phase respectively considering the respondents and recontacted samples.

Further, let $\lambda_{1}=n_{1}^{-1}\left(1-f_{1}\right)$ and $\lambda_{2}=n_{2}^{-1}\left(1-f_{2}\right)$ be sampling fractions for the first and second phase respectively, $\theta_{1}=n_{1}^{-1} W_{2}\left(k_{1}-1\right)$ and $\theta_{2}=n_{2}^{-1} W_{2}\left(k_{2}-\right.$ 1) are the sampling fractions for non-respondents who are recontacted at the first and second phase respectively, where $f_{1}=N^{-1} n_{1}$ and $f_{2}=N^{-1} n_{2}$. Also, $\lambda_{3}=\lambda_{2}-\lambda_{1}$ and $\theta_{3}=\theta_{2}-\theta_{1}$.

\section{Generalized class of regression-cum-ratio-exponential estimators}

Mohanty (1967) introduced a concept of combining regression and ratio estimators in simple random sampling and call it a regression-cum-ratio estimator. Similarly regression can be combined with exponential estimator. The sum of the ratio and exponential with some suitable weights can be multiplied with regression and it can help to see the role of the exponential part along with the ratio and regression parts. Also the rationale of suggesting the following general class of estimators is to search for the best member from all members of the class. Combining the regression, ratio and exponential parts, the class can be suggested as:

$$
\begin{aligned}
t_{q}=\left(\bar{y}_{2}^{*}+a \sum_{i=1}^{q_{1}} \alpha_{i}\left(\bar{x}_{1 i}^{*}-\bar{x}_{2 i}^{*}\right)\right) & {\left[b \prod_{i=q_{1}+1}^{q_{2}}\left(\frac{\bar{x}_{1 i}^{*}}{\bar{x}_{2 i}^{*}}\right)^{c \alpha_{i}}\right.} \\
& \left.+d \exp \sum_{i=q_{2}+1}^{q_{3}} \alpha_{i}\left(\frac{g\left(\bar{x}_{2 i}^{*}-\bar{x}_{1 i}^{*}\right)}{\left(\bar{x}_{2 i}^{*}+\bar{x}_{1 i}^{*}\right)}\right)\right]
\end{aligned}
$$

where $a, b, c, d$ and $g$ are suitable constants to be chosen for generating members of this class such that $b+d=1$; and $\alpha_{i}$ 's a are unknown constants to be determined by minimizing the mean square error of $t_{q}$ given in (4.1).

From Hansen and Hurwitz (1946), let $\bar{e}_{\bar{y}_{2}^{*}}=\bar{y}_{2}^{*}-\bar{Y}, \bar{e}_{x_{1 i}^{*}}=x_{1 i}^{*}-\bar{X}_{i}$ and $\bar{e}_{x_{2 i}^{*}}=\bar{x}_{2 i}^{*}-\bar{X}_{i}$ be the sampling errors of $y, i$ th auxiliary variable in the first phase and $i$ th auxiliary variable in the second phase respectively. Then simplifying and using binomial expansion, we can write (4.1) after ignoring the third and higher order terms as:

$$
\begin{aligned}
t_{q} \approx & \left(\bar{Y}+\bar{e}_{y_{2}^{*}}+a \sum_{i=1}^{q_{1}} \alpha_{i}\left(\bar{e}_{x_{1 i}^{*}}-\bar{e}_{x_{2 i}^{*}}\right)\right) \\
\times & {\left[b \prod _ { i = q _ { 1 } + 1 } ^ { q _ { 2 } } \left(1+c \alpha_{i} \frac{\bar{e}_{x_{1 i}^{*}}-\bar{e}_{x_{2 i}^{*}}}{\bar{X}_{i}}+\frac{c \alpha_{i}\left(c \alpha_{i}+1\right)}{2 \bar{X}_{i}^{2}} \bar{e}_{x_{2 i}^{*}}^{2}\right.\right.} \\
& \left.+\frac{c \alpha_{i}\left(c \alpha_{i}-1\right)}{2 \bar{X}_{i}^{2}} \bar{e}_{x_{1 i}^{*}}^{2}-c^{2} \alpha_{i}^{2} \frac{\bar{e}_{x_{1 i}^{*}} \bar{e}_{x_{2 i}^{*}}}{\bar{X}_{i}^{2}}\right)
\end{aligned}
$$




$$
\begin{aligned}
& +d\left(1+\sum_{i=q_{2}+1}^{q_{3}} \alpha_{i} \frac{g}{2 \bar{X}_{i}}\left(\bar{e}_{x_{2 i}^{*}}-\bar{e}_{x_{1 i}^{*}}\right)-\sum_{i=q_{2}+1}^{q_{3}} \alpha_{i} \frac{g\left(\bar{e}_{x_{2 i}^{*}}^{2}-\bar{e}_{x_{1 i}^{*}}^{2}\right.}{4 \bar{X}_{i}^{2}}\right. \\
& \left.\left.+\left(\sum_{i=q_{2}+1}^{q_{3}} \alpha_{i}\right)^{2}\left(\frac{g^{2}}{8 \bar{X}_{i}^{2}}\left(\bar{e}_{x_{2 i}^{*}}^{2}+\bar{e}_{x_{q i}^{*}}^{2}-2 \bar{e}_{x_{2 i}^{*}} \bar{e}_{x_{1 i}^{*}}\right)\right)\right)\right]
\end{aligned}
$$

or

$$
\begin{aligned}
& t_{q} \approx\left(\bar{Y}+\bar{e}_{y_{2}^{*}}+a \sum_{i=1}^{q_{1}} \alpha_{i}\left(\bar{e}_{x_{1 i}^{*}}-\bar{e}_{x_{2 i}^{*}}\right)\right) \\
& \times\left[1+b c \sum_{i=q_{1}+1}^{q_{2}} \frac{\alpha_{i}}{\bar{X}_{i}}\left(\bar{e}_{x_{1 i}^{*}}-\bar{e}_{x_{2 i}^{*}}\right)+b c \sum_{i=q_{1}+1}^{q_{2}} \frac{\alpha_{i}\left(c \alpha_{i}+1\right)}{2 \bar{X}_{i}^{2}} \bar{e}_{x_{2 i}^{*}}^{2}\right. \\
& +d \sum_{i=q_{2}+1}^{q_{3}} \frac{g \alpha_{i}}{2 \bar{X}_{i}}\left(\bar{e}_{x_{2 i}^{*}}-\bar{e}_{x_{1 i}^{*}}\right)+b c \sum_{i=q_{1}+1}^{q_{2}} \alpha_{i} \frac{\left(c \alpha_{i}-1\right)}{2 \bar{X}_{i}^{2}} \bar{e}_{x_{1 i}^{*}}^{2} \\
& -b c^{2} \sum_{i=q_{1}+1}^{q_{2}} \alpha_{i}^{2} \frac{\bar{e}_{x_{1 i}^{*}} \bar{e}_{x_{2 i}^{*}}}{\bar{X}_{i}^{2}}-d \sum_{i=q_{2}+1}^{q_{3}} \alpha_{i} \frac{g\left(\bar{e}_{x_{2 i}^{*}}^{2}-\bar{e}_{x_{1 i}^{*}}^{2}\right)}{4 \bar{X}_{i}^{2}} \\
& \left.+d\left(\sum_{i=q_{2}+1}^{q_{3}} \alpha_{i}\right)^{2}\left(\frac{g^{2}}{8 \bar{X}_{i}^{2}}\left(\bar{e}_{x_{2 i}^{*}}^{2}+\bar{e}_{x_{1 i}^{*}}^{2}-2^{b} \operatorname{are}_{x_{2 i}^{*}} \bar{e}_{x_{1 i}^{*}}\right)\right)\right]
\end{aligned}
$$

Again ignoring third and higher order terms for each expansion of the product and simplifying, we can write the expression as:

$$
\begin{aligned}
& t_{q} \approx \bar{Y}+\bar{e}_{y_{2}^{*}}+a \sum_{i=1}^{q_{1}} \alpha_{i}\left(\bar{e}_{x_{1 i}^{*}}-\bar{e}_{x_{2 i}^{*}}\right)+\bar{Y} b c \sum_{i=q_{1}+1}^{q_{2}} \frac{\alpha_{i}}{\bar{X}_{i}}\left(\bar{e}_{x_{1 i}^{*}}-\bar{e}_{x_{2 i}^{*}}\right) \\
& +\bar{Y} d \sum_{i=q_{1}+1}^{q_{3}} \alpha_{i} \frac{g}{2 \bar{X}_{i}}\left(\bar{e}_{x_{2 i}^{*}}-\bar{e}_{x_{1 i}^{*}}\right) \\
& +a b c \sum_{i=q_{1}+1}^{q_{2}} \frac{\alpha_{i}}{\bar{X}_{i}}\left(\bar{e}_{x_{1 i}^{*}}-\bar{e}_{x_{2 i}^{*}}\right) \sum_{i=1}^{q_{1}} \alpha_{i}\left(\bar{e}_{x_{1 i}^{*}}-\bar{e}_{x_{2 i}^{*}}\right) \\
& +\bar{Y} b c \sum_{i=q_{1}+1}^{q_{2}} \alpha_{i} \frac{\bar{e}_{x_{2 i}^{*}}^{2}}{2 \bar{X}_{i}^{2}}+\bar{Y} b c^{2} \sum_{i=q_{1}+1}^{q_{2}} \alpha_{i}^{2} \frac{\bar{e}_{x_{1 i}^{*}}^{2}}{2 \bar{X}_{i}^{2}}-\bar{Y} b c \sum_{i=q_{1}+1}^{q_{2}} \alpha_{i} \frac{\bar{e}_{x_{1 i}^{*}}^{2}}{2 \bar{X}_{i}^{2}} \\
& \left.-\bar{Y} b c^{2} \sum_{i=q_{1}+1}^{q_{2}} \alpha_{i}^{2} \frac{\bar{e}_{x_{1 i}^{*}} \bar{e}_{x_{2 i}^{*}}}{\bar{X}_{i}^{2}}-2 \bar{Y} d \frac{g^{2}}{8 \bar{X}_{i}^{2}}\left(\sum_{i=q_{2}+1}^{q_{3}} \alpha_{i}\right)\right)^{2} \bar{e}_{x_{2 i}^{*}} \bar{e}_{x_{1 i}^{*}} \\
& +\bar{Y} d g \sum_{i=q_{2}+1}^{q_{3}} \alpha_{i} \frac{\bar{e}_{x_{1 i}^{*}}}{4 \bar{X}_{i}^{2}}+\bar{Y} d \frac{g^{2}}{8 \bar{X}_{i}^{2}}\left(\sum_{i=q_{2}+1}^{q_{3}} \alpha_{i}\right)^{2} \bar{e}_{x_{1 i}^{*}}^{2} \\
& -\bar{Y} d g \sum_{i=q_{2}+1}^{q_{3}} \alpha_{i} \frac{\bar{e}_{x_{2 i}^{*}}^{2}}{4 \bar{X}_{i}^{2}}+\bar{Y} b c^{2} \sum_{i=q_{1}+1}^{q_{2}} \alpha_{i}^{2} \frac{\bar{e}_{x_{2 i}^{*}}^{2}}{2 \bar{X}_{i}^{2}}+b c \sum_{i=q_{1}+1}^{q_{2}} \alpha_{i} \frac{\bar{e}_{x_{1 i}^{*}}-\bar{e}_{x_{2 i}^{*}}}{\bar{X}_{i}} \bar{e}_{y_{2}^{*}}
\end{aligned}
$$




$$
\begin{aligned}
& +d \sum_{i=q_{2}+1}^{q_{3}} \alpha_{i} \frac{g}{2 \bar{X}_{i}}\left(\bar{e}_{x_{2 i}^{*}}-\bar{e}_{x_{1 i}^{*}}\right) \bar{e}_{y_{2}^{*}} \\
& +d \sum_{i=q_{2}+1}^{q_{3}} \alpha_{i} \frac{g}{2 \bar{X}_{i}}\left(\bar{e}_{x_{2 i}^{*}}-\bar{e}_{x_{1 i}^{*}}\right) a \sum_{i=1}^{q_{1}} \alpha_{i}\left(\bar{e}_{x_{1 i}^{*}}-\bar{e}_{x_{2 i}^{*}}\right) .
\end{aligned}
$$

We can write the above expression in matrix notation as:

$$
\begin{aligned}
& t_{q}-\bar{Y} \approx \bar{e}_{y_{2}^{*}}-a \boldsymbol{\alpha}_{q_{1}}^{t} \boldsymbol{d}_{q_{1}}^{*}-\bar{Y} b c \boldsymbol{\alpha}_{q_{2}}^{t} \boldsymbol{X}_{q_{2}} \boldsymbol{d}_{q_{2}}^{*} \\
& +\frac{\bar{Y}}{2} d g \boldsymbol{\alpha}_{q_{3}}^{t} \boldsymbol{X}_{q_{3}} \boldsymbol{d}_{q_{3}}^{*}+\frac{\bar{Y}}{2} b c^{2} \boldsymbol{\alpha}_{q_{2}}^{t} \boldsymbol{X}_{q_{2}}^{2} \boldsymbol{\alpha}_{q_{2}} \mathbf{1}_{q_{2}}^{t} \overline{\boldsymbol{e}}_{x_{2_{2}}^{*}}^{2} \\
& +\frac{\bar{Y}}{2} b c \boldsymbol{\alpha}_{q_{2}}^{t} \boldsymbol{X}_{q_{2}}^{2} \overline{\boldsymbol{e}}_{x_{q_{2}}^{*}}^{2}+\frac{\bar{Y}}{2} b c^{2} \boldsymbol{\alpha}_{q_{2}}^{t} \boldsymbol{X}_{q_{2}}^{2} \boldsymbol{\alpha}_{q_{2}} \mathbf{1}_{q_{2}}^{t} \overline{\boldsymbol{e}}_{x_{q_{2}}^{*}}^{2} \\
& -\bar{Y} b c^{2} \boldsymbol{\alpha}_{q_{2}}^{t} \boldsymbol{X}_{q_{2}}^{2} \overline{\boldsymbol{e}}_{x_{1_{2}}^{*}} \overline{\boldsymbol{e}}_{x_{2_{2}}^{*}}^{t} \boldsymbol{\alpha}_{q_{2}}-\frac{\bar{Y}}{2} b c \boldsymbol{\alpha}_{q_{2}}^{t} \boldsymbol{X}_{q_{2}}^{2} \overline{\boldsymbol{e}}_{x_{1_{q_{2}}}^{*}}^{2} \\
& -b c \boldsymbol{\alpha}_{q_{2}}^{t} \boldsymbol{X}_{q_{2}} \boldsymbol{d}_{q_{2}}^{*} \bar{e}_{y_{2}^{*}}+a b c \boldsymbol{\alpha}_{q_{2}}^{t} \boldsymbol{X}_{q_{2}} \boldsymbol{d}_{q_{2}}^{*} \boldsymbol{d}_{q_{1}}^{* t} \boldsymbol{\alpha}_{q_{1}} \\
& -\frac{\bar{Y}}{4} d g \boldsymbol{\alpha}_{q_{3}}^{t} \boldsymbol{X}_{q_{3}}^{2} \overline{\boldsymbol{e}}_{x_{2_{q_{3}}}^{*}}^{2}+\frac{\bar{Y}}{4} d g \boldsymbol{\alpha}_{q_{3}}^{t} \boldsymbol{X}_{q_{3}}^{2} \overline{\boldsymbol{e}}_{x_{1_{q_{3}}}^{*}}^{2}+\frac{d g}{2} \boldsymbol{\alpha}_{q_{3}}^{t} \boldsymbol{X}_{q_{3}} \boldsymbol{d}_{q_{3}}^{*} \bar{e}_{y_{2}^{*}} \\
& +\frac{\bar{Y}}{8} d g^{2} \boldsymbol{\alpha}_{q_{3}}^{t} X_{q_{3}}^{2} \boldsymbol{\alpha}_{q_{3}} \mathbf{1}_{q_{3}}^{t} \overline{\boldsymbol{e}}_{x_{q_{3}}^{*}}^{2}+\frac{\bar{Y}}{8} d g^{2} \boldsymbol{\alpha}_{q_{3}}^{t} \boldsymbol{X}_{q_{3}}^{2} \boldsymbol{\alpha}_{q_{3}} \mathbf{1}_{q_{3}}^{t} \overline{\boldsymbol{e}}_{x_{q_{3}}^{*}}^{2} \\
& -\frac{\bar{Y}}{4} d g^{2} \boldsymbol{\alpha}_{q_{3}}^{t} \boldsymbol{X}_{q_{3}}^{2} \overline{\boldsymbol{e}}_{x_{1_{q_{3}}}} \overline{\boldsymbol{e}}_{x_{1_{q_{3}}}^{*}}^{t} \boldsymbol{\alpha}_{q_{3}}-\frac{a d g}{2} \boldsymbol{\alpha}_{q_{3}}^{t} \boldsymbol{X}_{q_{3}} \boldsymbol{d}_{q_{3}}^{*} \boldsymbol{d}_{q_{1}}^{* t} \boldsymbol{\alpha}_{q_{1}} .
\end{aligned}
$$

where $\boldsymbol{X}_{q_{1}}=\left[\bar{X}_{i}^{-1}\right]_{\left(q_{1} \times q_{1}\right)} ; i=1, \ldots, q_{1}, \boldsymbol{X}_{q_{2}}=\left[\bar{X}_{i}^{-1}\right]_{\left(q_{2} \times q_{2}\right)} ; i=q_{1}+1, q_{1}+$ $2, \ldots, q_{2}$ and $\boldsymbol{X}_{q_{3}}=\left[\bar{X}_{i}^{-1}\right]_{\left(q_{3} \times q_{3}\right)} ; i=q_{2}+1, q_{2}+2, \ldots, q_{3}$ are the diagonal matrices and $q=q_{1}+q_{2}+q_{3} . \alpha_{q_{l}}$ 's a are vectors of unknown coefficient where $l=1,2,3$ and the vector $d_{q_{l} \times 1}^{* t}=\left[d_{i}^{*}\right]_{q_{l} \times 1}$, with $d_{i}^{*}=\left(\bar{e}_{x_{(2) i}}^{*}-\bar{e}_{x_{(1) i}}^{*}\right)$.

Again ignoring the second and higher order terms for obtaining the MSE to the terms of $o(1 / n)$, we have

$$
t_{q}-\bar{Y} \approx \bar{e}_{y_{2}^{*}}-a \boldsymbol{\alpha}_{q_{1}}^{t} \boldsymbol{d}_{q_{1}}^{*}-\bar{Y} b c \boldsymbol{\alpha}_{q_{2}}^{t} \boldsymbol{X}_{q_{2}} \boldsymbol{d}_{q_{2}}^{*}+\frac{\bar{Y}}{2} d g \boldsymbol{\alpha}_{q_{3}}^{t} \boldsymbol{X}_{q_{3}} \boldsymbol{d}_{q_{3}}^{*} .
$$

Squaring and taking expectation, we have

$$
\operatorname{MSE}\left(t_{q}\right)=E\left[\bar{e}_{\bar{y}_{2}^{*}}-\boldsymbol{h}_{q}^{t} \boldsymbol{m}_{q}\right]^{2}
$$

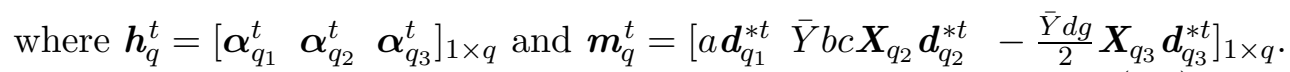

To find the optimum value of $\boldsymbol{h}$, differentiating the equation (4.3) w.r.t. $\boldsymbol{h}$ and equating to zero, we get:

$$
2 \boldsymbol{m}_{q} E\left[\bar{e}_{\bar{y}_{2}^{*}}-\boldsymbol{m}_{q}^{t} \boldsymbol{h}_{q}\right]=0
$$

or

$$
E\left(\boldsymbol{m}_{q} \bar{e}_{\bar{y}_{2}^{*}}\right)-E\left(\boldsymbol{m}_{q} \boldsymbol{m}_{q}^{t}\right) \boldsymbol{h}_{q}=0
$$


or

$$
E\left(\boldsymbol{m}_{q}\right)-E\left(\boldsymbol{\Lambda}_{q}\right) \boldsymbol{h}_{q}=0
$$

where

$$
E(\boldsymbol{\omega})=\boldsymbol{\Phi}_{y x_{q}}=e\left[\begin{array}{c}
a \boldsymbol{d}_{q_{1}}^{*} \bar{e}_{y_{2}^{*}} \\
\bar{Y} b c \boldsymbol{X}_{q_{2}} \boldsymbol{d}_{q_{2}}^{*} \bar{e}_{y_{2}^{*}} \\
-\frac{\bar{Y} d g}{2} \boldsymbol{X}_{q_{3}} \boldsymbol{d}_{q_{3}}^{*} \bar{e}_{y_{2}^{*}}
\end{array}\right]_{q \times 1}=\left[\begin{array}{c}
a \boldsymbol{\Phi}_{y x_{q_{1}}} \\
\bar{Y} b c \boldsymbol{X}_{q_{2}} \boldsymbol{\Phi}_{y x_{q_{2}}} \\
-\bar{Y} d g \\
-\frac{\boldsymbol{X}_{q_{3}}}{\boldsymbol{\Phi}_{y x_{q_{3}}}}
\end{array}\right]_{q \times 1}
$$

and

$$
\begin{aligned}
E\left(\boldsymbol{\Lambda}_{q}\right) & =\boldsymbol{T}_{q} \\
& =E\left[\begin{array}{ccc}
a^{2} \boldsymbol{d}_{q_{1}}^{*} \boldsymbol{d}_{q_{1}}^{* t} & \bar{Y} a b c \boldsymbol{d}_{q_{1}}^{*} \boldsymbol{d}_{q_{2}}^{* t} \boldsymbol{X}_{q_{2}} & -\frac{\bar{Y} a d g}{2} \boldsymbol{d}_{q_{1}}^{*} \boldsymbol{d}_{q_{3}}^{* t} \boldsymbol{X}_{q_{3}} \\
& \bar{Y}^{2} b^{2} c^{2} \boldsymbol{X}_{q_{2}} \boldsymbol{d}_{q_{2}}^{*} \boldsymbol{d}_{q_{2}}^{* t} \boldsymbol{X}_{q_{2}} & -\frac{\bar{Y}^{2} b c d g}{2} \boldsymbol{X}_{q_{2}} \boldsymbol{d}_{q_{2}}^{*} \boldsymbol{d}_{q_{3}}^{* t} \boldsymbol{X}_{q_{3}} \\
& \frac{\bar{Y}^{2} d^{2} g^{2}}{4} \boldsymbol{X}_{q_{3}} \boldsymbol{d}_{q_{3}}^{*} \boldsymbol{d}_{q_{3}}^{* t} \boldsymbol{X}_{q_{3}}
\end{array}\right]_{q \times q}
\end{aligned}
$$

or

$$
\boldsymbol{T}_{q}=\left[\begin{array}{ccc}
a^{2} \boldsymbol{\Lambda}_{q_{1}} & \bar{Y} a b c \boldsymbol{\Lambda}_{q_{12}} \boldsymbol{X}_{q_{2}} & -\frac{\bar{Y} a d g}{2} \boldsymbol{\Lambda}_{q_{13}} \boldsymbol{X}_{q_{3}} \\
\bar{Y}^{2} b^{2} c^{2} \boldsymbol{X}_{q_{2}} \boldsymbol{\Lambda}_{q_{2}} \boldsymbol{X}_{q_{2}} & -\frac{\bar{Y}^{2} b c d g}{\bar{Y}^{2} \bar{d}^{2} g^{2}} \boldsymbol{X}_{q_{2}} \boldsymbol{\Lambda}_{q_{23}} \boldsymbol{X}_{q_{3}} \\
& \frac{\boldsymbol{X}_{q_{3}} \boldsymbol{\Lambda}_{q_{3}} \boldsymbol{X}_{q_{3}}}{4}
\end{array}\right]
$$

where

$$
E\left(\boldsymbol{d}_{q_{l}} e_{\bar{y}_{2}^{*}}\right)=\left[\lambda_{3} S_{y x_{i}}+\theta_{3} S_{y x_{i 2}}\right]_{q_{l} \times 1}=\boldsymbol{\Phi}_{y x_{q l}},
$$

and

$$
E\left(\boldsymbol{d}_{q_{l}} \boldsymbol{d}_{q_{l}}^{t}\right)=\left[\lambda_{3} S_{x_{i} x_{j}}+\theta S_{x_{i} x_{j(2)}}\right]_{q_{l} \times q_{l}}=\boldsymbol{\Lambda}_{q_{l}} ; \quad i=1,2, \ldots, q_{l}, \quad l=1,2,3 .
$$

Now (4.4) can be written as

$$
\boldsymbol{\Phi}_{y x_{q}}-\boldsymbol{T}_{q} \boldsymbol{h}_{q}=0
$$

or

$$
\boldsymbol{h}_{q}=\boldsymbol{T}_{q}^{-1} \boldsymbol{\Phi}_{y x_{q}}, \text { provided } \boldsymbol{T}_{q}^{-1} \text { exist. }
$$

To use the members of the suggested class practically, we require the elements of $\boldsymbol{h}$. In almost all practical situations, the values of unknown parameters required for $\boldsymbol{h}$ are not available, hence for practical purposes we have to estimate 
the elements of $\boldsymbol{h}$ from sample observations. Without knowing or estimating the elements, we cannot use the members of the proposed class.

Now, from (4.2), ignoring the third and higher order terms for each expansion of the product, we get

$$
\begin{aligned}
& E\left(t_{1}^{m}-\bar{Y}\right) \approx E\left(\bar{e}_{y_{2}^{*}}-a \boldsymbol{\alpha}_{q_{1}}^{t} \boldsymbol{d}_{q_{1}}^{*}-\bar{Y} b c \boldsymbol{\alpha}_{q_{2}}^{t} \boldsymbol{X}_{q_{2}} \boldsymbol{d}_{q_{2}}^{*}+\frac{\bar{Y}}{2} d g \boldsymbol{\alpha}_{q_{3}}^{t} \boldsymbol{X}_{q_{3}} \boldsymbol{d}_{q_{3}}^{*}\right. \\
& +\frac{\bar{Y}}{2} b c^{2} \boldsymbol{\alpha}_{q_{2}}^{t} \boldsymbol{X}_{q_{2}}^{2} \boldsymbol{\alpha}_{q_{2}} \mathbf{1}_{q_{2}}^{t} \overline{\boldsymbol{e}}_{x_{2_{2}}^{*}}^{2}+\frac{\bar{Y}}{2} b c \boldsymbol{\alpha}_{q_{2}}^{t} \boldsymbol{X}_{q_{2}}^{2} \overline{\boldsymbol{e}}_{x_{q_{2}}^{*}}^{2} \\
& +\frac{\bar{Y}}{2} b c^{2} \boldsymbol{\alpha}_{q_{2}}^{t} \boldsymbol{X}_{q_{2}}^{2} \boldsymbol{\alpha}_{q_{2}} \mathbf{1}_{q_{2}}^{t} \overline{\boldsymbol{e}}_{x_{q_{2}}^{*}}^{2}-\bar{Y} b c^{2} \boldsymbol{\alpha}_{q_{2}}^{t} \boldsymbol{X}_{q_{2}}^{2} \overline{\boldsymbol{e}}_{x_{1_{2}}^{*}} \overline{\boldsymbol{e}}_{x_{2_{2}}^{*}}^{t} \boldsymbol{\alpha}_{q_{2}} \\
& -\frac{\bar{Y}}{2} b c \boldsymbol{\alpha}_{q_{2}}^{t} \boldsymbol{X}_{q_{2}}^{2} \overline{\boldsymbol{e}}_{x_{1_{2}}^{*}}^{2}-b c \boldsymbol{\alpha}_{q_{2}}^{t} \boldsymbol{X}_{q_{2}} \boldsymbol{d}_{q_{2}}^{*} \bar{e}_{y_{2}^{*}} \\
& +a b c \boldsymbol{\alpha}_{q_{2}}^{t} \boldsymbol{X}_{q_{2}} \boldsymbol{d}_{q_{2}}^{*} \boldsymbol{d}_{q_{1}}^{* \prime} \boldsymbol{\alpha}_{q_{1}}-\frac{\bar{Y}}{4} d g \boldsymbol{\alpha}_{q_{3}}^{t} \boldsymbol{X}_{q_{3}}^{2} \overline{\boldsymbol{e}}_{x_{2_{3}}^{*}}^{2} \\
& +\frac{\bar{Y}}{4} d g \boldsymbol{\alpha}_{q_{3}}^{t} \boldsymbol{X}_{q_{3}}^{2} \overline{\boldsymbol{e}}_{x_{1_{3}}^{*}}^{2}+\frac{\bar{Y}}{8} d g^{2} \boldsymbol{\alpha}_{q_{3}}^{t} \boldsymbol{X}_{q_{3}}^{2} \boldsymbol{\alpha}_{q_{3}} \mathbf{1}_{q_{3}}^{t} \overline{\boldsymbol{e}}_{x_{q_{3}}^{*}}^{2} \\
& +\frac{\bar{Y}}{8} d g^{2} \boldsymbol{\alpha}_{q_{3}}^{t} \boldsymbol{X}_{q_{3}}^{2} \boldsymbol{\alpha}_{q_{3}} \mathbf{1}_{q_{3}}^{t} \overline{\boldsymbol{e}}_{x_{1_{3}}^{*}}^{2}-\frac{\bar{Y}}{4} d g^{2} \boldsymbol{\alpha}_{q_{3}}^{t} \boldsymbol{X}_{q_{3}}^{2} \overline{\boldsymbol{e}}_{x_{1_{3}}^{*}} \overline{\boldsymbol{e}}_{x_{1_{3}}^{*}}^{t} \boldsymbol{\alpha}_{q_{3}} \\
& \left.-\frac{a d g}{2} \boldsymbol{\alpha}_{q_{3}}^{t} \boldsymbol{X}_{q_{3}} \boldsymbol{d}_{q_{3}}^{*} \boldsymbol{d}_{q_{1}}^{* t} \boldsymbol{\alpha}_{q_{1}}+\frac{d g}{2} \boldsymbol{\alpha}_{q_{3}}^{t} \boldsymbol{X}_{q_{3}} \boldsymbol{d}_{q_{3}}^{*} \bar{e}_{y_{2}^{*}}\right) \text {. }
\end{aligned}
$$

Now taking expectation, bias can be written as:

$$
\begin{aligned}
& \operatorname{Bias}\left(t_{q}\right) \approx b c\left\{a \boldsymbol{\alpha}_{q_{2}}^{t} \boldsymbol{X}_{q_{2}} \boldsymbol{\Lambda}_{q_{21}} \boldsymbol{\alpha}_{q_{1}}-\boldsymbol{\alpha}_{q_{2}}^{t} \boldsymbol{X}_{q_{2}} \boldsymbol{\Phi}_{y x_{q_{2}}}+\frac{\bar{Y}}{2} \boldsymbol{\alpha}_{q_{2}}^{t} \boldsymbol{X}_{q_{2}}^{2}\left(s_{2_{q_{2}}}^{*}-\boldsymbol{s}_{1_{q_{2}}}^{*}\right)\right. \\
&\left.+c\left(\frac{\bar{Y}}{2} \boldsymbol{\alpha}_{q_{2}}^{t} \boldsymbol{X}_{q_{2}}^{2} \boldsymbol{\alpha}_{q_{2}} \mathbf{1}_{q_{2}}\left(\boldsymbol{s}_{1_{q_{2}}}^{*}+\boldsymbol{s}_{2_{q_{2}}}^{*}\right)-\bar{Y} \boldsymbol{\alpha}_{q_{2}}^{t} \boldsymbol{X}_{q_{2}}^{2} \boldsymbol{\Sigma}_{q_{2}} \boldsymbol{\alpha}_{q_{2}}\right)\right\} \\
&+d g\{-\frac{a}{2} \boldsymbol{\alpha}_{q_{3}}^{t} \boldsymbol{X}_{q_{3}} \boldsymbol{\Lambda}_{3_{q_{3}}} \boldsymbol{\alpha}_{q_{1}}+\frac{\bar{Y}}{4} \boldsymbol{\alpha}_{q_{3}}^{t} \boldsymbol{X}_{q_{3}}^{2}\left(\boldsymbol{s}_{1_{q_{3}}}^{*}-\boldsymbol{s}_{2_{q_{3}}}^{*}\right) \\
&+\frac{1}{2} \boldsymbol{\alpha}_{q_{3}}^{t} \boldsymbol{X}_{q_{3}} \boldsymbol{\Phi}_{y x_{q_{3}}}+g\left(\frac{\bar{Y}}{8} \boldsymbol{\alpha}_{q_{3}}^{t} \boldsymbol{X}_{q_{3}}^{2} \boldsymbol{\alpha}_{q_{3}} \mathbf{1}_{q_{3}}^{t}\left(s_{1_{q_{3}}}^{*}+\boldsymbol{s}_{q_{q_{3}}}^{*}\right)\right. \\
&\left.\left.-\frac{\bar{Y}}{4} \boldsymbol{\alpha}_{q_{3}}^{t} \boldsymbol{X}_{q_{3}}^{2} \boldsymbol{\Sigma}_{q_{3}} \boldsymbol{\alpha}_{q_{3}}\right)\right\}
\end{aligned}
$$

$\operatorname{Bias}\left(t_{q}\right)=$ Bias due to regression-cum-ratio + Bias due to regression-cum-exponential,

where the values of $\alpha_{q_{l}}$ 's can be used from $\boldsymbol{h}$ given in (4.7) and

$$
\begin{aligned}
& E\left(\overline{\boldsymbol{e}}_{x_{1_{q_{l}}}^{*}} \overline{\boldsymbol{e}}_{x_{2_{q_{l}}}^{*}}^{t}\right)=E\left(\overline{\boldsymbol{e}}_{x_{1_{q_{l}}}^{*}} \overline{\boldsymbol{e}}_{x_{1_{q_{l}}}^{*}}^{t}\right)=\left[\lambda_{1} S_{x_{i} x_{j(2)}}\right]_{q_{l} \times q_{l}}=\boldsymbol{\Sigma}_{q_{l}}, \\
& E\left(\overline{\boldsymbol{e}}_{x_{1_{q_{l}}}^{*}}^{2}\right)=\left[\lambda_{1} S_{x_{i}}^{2}+\theta_{1} S_{x_{i}(2)}^{2}\right]_{q_{l} \times 1}=s_{1_{q_{l}}}^{*}
\end{aligned}
$$


and

$$
E\left(\overline{\boldsymbol{e}}_{x_{2_{l}}^{*}}^{2}\right)=\left[\lambda_{2} S_{x_{i}}^{2}+\theta_{2} S_{x_{i}(2)}^{2}\right]_{q_{l} \times 1}=\boldsymbol{s}_{2_{q_{l}}}^{*} .
$$

By using the normal equations that are used to find the optimum values given in equation (4.7), equation (4.3) can be written as:

$$
\operatorname{MSE}\left(t_{q}\right)=E\left(\bar{e}_{y_{2}^{*}}\left(\bar{e}_{y_{2}^{*}}-\boldsymbol{h}_{q}^{t} \boldsymbol{m}_{q}\right)\right)
$$

or

$$
\operatorname{MSE}\left(t_{q}\right)=E\left(\bar{e}_{y_{2}^{*}}^{2}\right)-\boldsymbol{h}_{q}^{t} E\left(\boldsymbol{m}_{q} \bar{e}_{y_{2}^{*}}\right)
$$

or

$$
\operatorname{MSE}\left(t_{q}\right)=\lambda_{2} S_{y}^{2}+\theta S_{y_{(2)}}^{2}-\boldsymbol{h}_{q}^{t} \boldsymbol{\Phi}_{y x_{q}} .
$$

Now, using equation (4.5), we get:

$$
\operatorname{MSE}\left(t_{q}\right)=\lambda_{2} S_{y}^{2}+\theta S_{y_{(2)}}^{2}-\boldsymbol{\Phi}_{y x_{q}}^{t} \boldsymbol{T}_{q}^{-1} \boldsymbol{\Phi}_{y x_{q}} .
$$

A number of special cases of proposed class (4.1) may be deduced. These members of the class with their expressions of bias and MSE's are given in the following remarks.

Remark 1 . We can obtain a regression estimator using multi-auxiliary variables when non-response occurs at both phases by substituting $a=1, b=c=$ $d=g=0$ in (4.1) i.e.

$$
t_{q_{\mathrm{reg}}}=\bar{y}_{2}^{*}+\sum_{i=1}^{q_{1}} \alpha_{1_{i}}\left(\bar{x}_{1 i}^{*}-\bar{x}_{2 i}^{*}\right) .
$$

The bias of (4.10) can be obtained by substituting $a=1, b=c=d=g=0$ in (4.8) as

$$
\operatorname{Bias}\left(t_{q_{\mathrm{reg}}}\right)=0 \text {. }
$$

The optimum value $\alpha_{1_{q_{1}}}=\left(\boldsymbol{\Lambda}_{q_{1}}\right)^{-1} \boldsymbol{\Phi}_{y x_{q_{1}}}$, the mean square error of (4.10) can be obtained by substituting $a=1, b=c=d=g=0$ in (4.5) and (4.6) and using (4.9) as

$$
\operatorname{MSE}\left(t_{q_{\mathrm{reg}}}\right)=\lambda_{2} S_{y}^{2}+\theta_{2} S_{y(2)}^{2}-\Phi_{y x_{q_{1}}}\left(\Lambda_{q_{1}}\right)^{-1} \Phi_{y x_{q_{1}}} .
$$

For $a=-1$, the difference estimator can be obtained as

$$
t_{q_{\mathrm{reg}}}=\bar{y}_{2}^{*}+\sum_{i=1}^{q_{1}} \alpha_{1_{i}}\left(\bar{x}_{2 i}^{*}-\bar{x}_{1 i}^{*}\right) .
$$

Now for the optimum value $\boldsymbol{\alpha}_{2_{q_{1}}}=-\boldsymbol{\alpha}_{1_{q_{1}}}$, the MSE will remain the same as in (4.11) and bias will be zero. 
Remark 2. We can obtain a ratio estimator by substituting $b=c=1$, $a=d=g=0$ in (4.1) i.e.

$$
t_{q_{\mathrm{ratio}}}=\bar{y}_{2}^{*} \prod_{i=1}^{q_{2}}\left(\frac{\bar{x}_{1 i}^{*}}{\bar{x}_{2 i}^{*}}\right)^{\alpha_{1_{i}}}
$$

The bias of (4.12) can be obtained by substituting $b=c=1, a=d=g=0$ in (4.8) as

$$
\begin{aligned}
\operatorname{Bias}\left(t_{q_{\text {ratio }}}\right) \approx & -\alpha_{1_{q_{2}}}^{t} X_{q_{2}} \Phi_{y x_{q_{2}}}+\frac{\bar{Y}}{2} \alpha_{1_{q_{2}}}^{t} X_{q_{2}}^{2}\left(s_{2_{q_{2}}}^{*}-s_{1_{q_{2}}}^{*}\right) \\
& +\frac{\bar{Y}}{2} \alpha_{1_{q_{2}}}^{t} X_{q_{2}}^{2} \alpha_{1_{q_{2}}} 1_{q_{2}}\left(s_{1_{q_{2}}}^{*}+s_{2_{q_{2}}}^{*}\right)-\bar{Y} \alpha_{1_{q_{2}}}^{t} X_{q_{2}}^{2} \Sigma_{q_{2}} \alpha_{1_{q_{2}}}
\end{aligned}
$$

For the optimum value $\boldsymbol{\alpha}_{1_{q_{2}}}=\bar{Y}^{-1} \boldsymbol{X}_{q_{2}}^{-1}\left(\boldsymbol{\Lambda}_{q_{2}}\right)^{-1} \boldsymbol{\Phi}_{y x_{q_{2}}}$, the mean square error of (4.12) can be obtained by substituting $b=c=1, a=d=g=0$ in (4.5) and (4.6) and using (4.9) as:

$$
\operatorname{MSE}\left(t_{q_{\mathrm{ratio}}}\right) \approx \lambda_{2} S_{y}^{2}+\theta_{2} S_{y_{2}}^{2}-\Phi_{y x_{q_{2}}}\left(\Lambda_{q_{2}}\right)^{-1} \Phi_{y x_{q_{2}}} .
$$

We can obtain a product estimator when $b=-1$ as

$$
t_{q_{\mathrm{prod}}}=\bar{y}_{2}^{*} \prod_{i=1}^{q_{2}}\left(\frac{\bar{x}_{2 i}^{*}}{\bar{x}_{1 i}^{*}}\right)^{\alpha_{2_{i}}}
$$

For the optimum value $\boldsymbol{\alpha}_{2_{q_{2}}}=-\boldsymbol{\alpha}_{1_{q_{2}}}$, the bias and MSE will remain the same as for the estimator given (4.12). If $q_{1}=q_{2},(4.13)$ will be the same as (4.11).

Remark 3. We can obtain an exponential-ratio estimator by substituting $d=g=-1, a=b=c=0$ in (4.1) i.e.

$$
t_{q_{e r}}=\bar{y}_{2}^{*} \exp \sum_{i=q_{2}+1}^{q_{3}} \alpha_{1_{i}}\left(\frac{\left(\bar{x}_{1 i}^{*}-\bar{x}_{2 i}^{*}\right)}{\left(\bar{x}_{2 i}^{*}+\bar{x}_{1 i}^{*}\right)}\right) .
$$

The bias of (4.14) can be obtained by substituting $d=g=-1, a=b=c=0$ in (4.8) as

$$
\begin{aligned}
\operatorname{Bias}\left(t_{q_{e r}}\right) \approx & \frac{\bar{Y}}{4} \boldsymbol{\alpha}_{q_{3}}^{t} \boldsymbol{X}_{q_{3}}^{2}\left(\boldsymbol{s}_{1_{q_{3}}}^{*}-\boldsymbol{s}_{2_{q_{3}}}^{*}\right)+\frac{1}{2} \boldsymbol{\alpha}_{q_{3}}^{t} \boldsymbol{X}_{q_{3}} \boldsymbol{\Phi}_{y x_{q_{3}}} \\
& +\frac{\bar{Y}}{8} \boldsymbol{\alpha}_{q_{3}}^{t} \boldsymbol{X}_{q_{3}}^{2} \boldsymbol{\alpha}_{q_{3}} \mathbf{1}_{q_{3}}^{t}\left(\boldsymbol{s}_{1_{q_{3}}}^{*}+\boldsymbol{s}_{2_{q_{3}}}^{*}\right)-\frac{\bar{Y}}{4} \boldsymbol{\alpha}_{q_{3}}^{t} \boldsymbol{X}_{q_{3}}^{2} \boldsymbol{\Sigma}_{q_{3}} \boldsymbol{\alpha}_{q_{3}}
\end{aligned}
$$

The optimum value $\boldsymbol{\alpha}_{1_{q_{3}}}=2 \bar{Y}^{-1} \boldsymbol{X}_{q_{3}}^{-1}\left(\boldsymbol{\Delta}_{q_{3}}\right)^{-1} \boldsymbol{\Phi}_{y x_{q_{3}}}$. The mean square error of (4.14) can be obtained by substituting $d=g=-1, a=b=c=0$ in (4.5) and (4.6) and using (4.9) as

$$
\operatorname{MSE}\left(t_{q_{e r}}\right)=\lambda_{2} S_{y}^{2}+\theta_{2} S_{y_{2}}^{2}-\Phi_{y x_{q_{2}}}\left(\Delta_{q_{2}}\right)^{-1} \Phi_{y x_{q_{2}}} .
$$


For $g=1$ we can obtain the exponential-product estimator

$$
t_{q_{e r}}=\bar{y}_{2}^{*} \exp \sum_{i=q_{2}+1}^{q_{3}} \alpha_{2_{i}}\left(\frac{\left(\bar{x}_{2 i}^{*}-\bar{x}_{1 i}^{*}\right)}{\left(\bar{x}_{2 i}^{*}+\bar{x}_{1 i}^{*}\right)}\right) .
$$

The optimum value $\boldsymbol{\alpha}_{2_{q_{3}}}=-\boldsymbol{\alpha}_{1_{q_{3}}}$, The bias MSE will remain the same as for the estimator given in (4.14).

Remark 4. We can obtain a regression-cum-ratio estimator by substituting $a=b=c=1, d=g=0$ in (4.1) i.e.

$$
t_{q_{r c r}}=\left\{\bar{y}_{2}^{*}+\sum_{i=1}^{q_{1}} \alpha_{3_{i}}\left(\bar{x}_{1 i}^{*}-\bar{x}_{2 i}^{*}\right)\right\}\left\{\prod_{i=1}^{q_{2}}\left(\frac{\bar{x}_{1 i}^{*}}{\bar{x}_{2 i}^{*}}\right)^{\alpha_{3 i}}\right\} .
$$

The bias of (4.16) can be obtained by substituting $a=b=c=1, d=g=0$ in (4.8) as

$$
\begin{aligned}
\operatorname{Bias}\left(t_{q_{r c r}}\right) \approx & \boldsymbol{\alpha}_{3_{q_{2}}}^{t} \boldsymbol{X}_{q_{2}} \boldsymbol{\Delta}_{q_{21}} \boldsymbol{\alpha}_{3_{q_{1}}}-\boldsymbol{\alpha}_{3_{q_{2}}}^{t} \boldsymbol{X}_{q_{2}} \boldsymbol{\Phi}_{y x_{q_{2}}}+\frac{\bar{Y}}{2} \boldsymbol{\alpha}_{3_{q_{2}}}^{t} \boldsymbol{X}_{q_{2}}^{2}\left(s_{2_{q_{2}}}^{*}-\boldsymbol{s}_{1_{q_{2}}}^{*}\right) \\
& +\frac{\bar{Y}}{2} \boldsymbol{\alpha}_{3_{q_{2}}}^{t} \boldsymbol{X}_{q_{2}}^{2} \boldsymbol{\alpha}_{3_{q_{2}}} \mathbf{1}_{q_{2}}^{t}\left(s_{1_{q_{2}}}^{*}+\boldsymbol{s}_{2_{q_{2}}}^{*}\right)-\bar{Y} \boldsymbol{\alpha}_{3_{q_{2}}}^{t} \boldsymbol{X}_{q_{2}}^{2} \boldsymbol{\Sigma}_{q_{2}} \boldsymbol{\alpha}_{3_{q_{2}}}
\end{aligned}
$$

The optimum values $\boldsymbol{\alpha}_{3_{1}}$ and $\boldsymbol{\alpha}_{3_{q_{2}}}$ are $\alpha_{3_{q_{1}}}=\boldsymbol{\Delta}_{q_{1}}^{-1} \boldsymbol{\Phi}_{y x_{q_{3}}}+\boldsymbol{\Delta}_{q_{1}}^{-1} \boldsymbol{\Delta}_{q_{12}} \boldsymbol{\Delta}_{q_{2}}^{*-1} \boldsymbol{\Delta}_{q_{21}}^{-1}$. $\boldsymbol{\Delta}_{q_{1}}^{-1} \boldsymbol{\Phi}_{y x_{q_{1}}}-\boldsymbol{\Delta}_{q_{1}}^{-1} \boldsymbol{\Delta}_{q_{12}} \boldsymbol{\Delta}_{q_{2}}^{*-1} \boldsymbol{\Phi}_{y x_{q_{1}}}$ and $\alpha_{q_{q_{2}}}=\bar{Y}^{-1} X_{q_{2}}^{-1} \boldsymbol{\Delta}_{q_{2}}^{*-1}\left(\boldsymbol{\Phi}_{y x_{q_{2}}}-\boldsymbol{\Delta}_{q_{21}} \boldsymbol{\Delta}_{q_{1}}^{-1}\right.$. $\left.\boldsymbol{\Phi}_{y x_{q_{1}}}\right)$, where $\boldsymbol{\Delta}_{q_{2}}^{*}=\left(\boldsymbol{\Delta}_{q_{2}}-\boldsymbol{\Delta}_{q_{21}} \boldsymbol{\Delta}_{q_{1}}^{-1} \boldsymbol{\Delta}_{q_{12}}\right)$.

The mean square error of (4.16) can be obtained by substituting $a=b=$ $c=1, d=g=0$ in (4.5) and (4.6) and using (4.9) as

$$
\begin{aligned}
\operatorname{MSE}\left(t_{q_{r c r}}\right) \approx & \lambda_{2} S_{y}^{2}+\theta_{2} S_{y_{2}}^{2}-\Phi_{y x_{q_{1}}}^{t} \Delta_{q_{1}}^{-1} \Phi_{y x_{q_{1}}} \\
& +\left(2 \Phi_{y x_{q_{2}}}^{t}-\Phi_{y x_{q_{1}}}^{t} B_{q_{12}}^{*}\right) \Delta_{q_{2}}^{*-1} B_{q_{21}}^{*} \Phi_{y x_{q_{1}}}^{t}-\Phi_{y x_{q_{2}}}^{t} \Delta_{q_{2}}^{*-1} \Phi_{y x_{q_{2}}}^{t}
\end{aligned}
$$

where $B_{\left(q_{12}\right)}^{*}=\Delta_{q_{1}}^{-1} \Delta_{q_{12}}$.

For $(a, b, c)=\{(1,1,-1),(-1,1,1),(-1,1,-1)\}$, the regression-cum-product, difference-cum-ratio and differencecum-product estimator are

$$
\begin{aligned}
& t_{q_{r c p}}=\left\{\bar{y}_{2}^{*}+\sum_{i=1}^{q_{1}} \alpha_{r_{i}}\left(\bar{x}_{1 i}^{*}-\bar{x}_{2 i}^{*}\right)\right\}\left\{\prod_{i=1}^{q_{2}}\left(\frac{\bar{x}_{2 i}^{*}}{\bar{x}_{1 i}^{*}}\right)^{\alpha_{4_{i}}}\right\}, \\
& t_{q_{d c r}}=\left\{\bar{y}_{2}^{*}+\sum_{i=1}^{q_{1}} \alpha_{5_{i}}\left(\bar{x}_{2 i}^{*}-\bar{x}_{1 i}^{*}\right)\right\}\left\{\prod_{i=1}^{q_{2}}\left(\frac{\bar{x}_{1 i}^{*}}{\bar{x}_{2 i}^{*}}\right)^{\alpha_{5_{i}}}\right\} .
\end{aligned}
$$

and $\left.t_{q_{d c p}}=\left\{\bar{y}_{2}^{*}+\sum_{i=1}^{q_{1}} \alpha_{6_{i}}\left(\bar{x}_{2 i}^{*}-\bar{x}_{1 i}^{*}\right)\right\}\left\{\prod_{i=1}^{q_{2}}\left(\bar{x}_{2 i}^{*}\right)^{\alpha_{1 i}}\right)^{\alpha_{i}}\right\}$ respectively. The optimum values for $t_{q_{r c p}}$ are $\boldsymbol{\alpha}_{4_{q_{1}}}=\boldsymbol{\alpha}_{q_{q_{1}}}$ and $\boldsymbol{\alpha}_{4_{q_{2}}}=-\boldsymbol{\alpha}_{3_{q_{2}}}$, for $t_{m_{d c r}} \boldsymbol{\alpha}_{5_{q_{1}}}=-\boldsymbol{\alpha}_{3_{q_{1}}}$ and $\boldsymbol{\alpha}_{5_{q_{2}}}=\boldsymbol{\alpha}_{3_{q_{2}}}$ and for $t_{q_{d c p}} \boldsymbol{\alpha}_{6_{q_{1}}}=-\boldsymbol{\alpha}_{3_{q_{1}}}$ and $\boldsymbol{\alpha}_{6_{q_{2}}}=-\boldsymbol{\alpha}_{3_{q_{2}}}$.

The bias and MSE of regression-cum-product, difference-cum-ratio and difference-cum-product estimators are the same as for the estimator given in (4.16). 
Remark 5. We can obtain a regression-cum-exponential-ratio estimator by substituting $a=d=g=-1, b=c=0$ in (4.1) i.e.

$$
t_{q_{r c e r}}=\left\{\bar{y}_{2}^{*}+\sum_{i=1}^{q_{1}} \alpha_{7_{i}}\left(\bar{x}_{1 i}^{*}-\bar{x}_{2 i}^{*}\right)\right\}\left\{\exp \sum_{i=q_{2}+1}^{q_{3}} \alpha_{7_{i}}\left(\frac{\left(\bar{x}_{1 i}^{*}-\bar{x}_{2 i}^{*}\right)}{\left(\bar{x}_{2 i}^{*}+\bar{x}_{1 i}^{*}\right)}\right)\right\} .
$$

The bias of (4.18) can be obtained by substituting $a=d=g=-1, b=c=0$ in (4.8) as

$$
\begin{aligned}
\operatorname{Bias}\left(t_{q_{r c e r}}\right) \approx & -\frac{1}{2} \boldsymbol{\alpha}_{q_{3}}^{t} \boldsymbol{X}_{q_{3}} \boldsymbol{\Delta}_{3_{q_{31}}} \boldsymbol{\alpha}_{q_{1}}+\frac{\bar{Y}}{4} \boldsymbol{\alpha}_{q_{3}}^{t} \boldsymbol{X}_{q_{3}}^{2}\left(\boldsymbol{s}_{1_{q_{3}}}^{*}-\boldsymbol{s}_{2_{q_{3}}}^{*}\right)+\frac{1}{2} \boldsymbol{\alpha}_{q_{3}}^{t} \boldsymbol{X}_{q_{3}} \boldsymbol{\Phi}_{y_{q_{3}}} \\
& +\frac{\bar{Y}}{8} \boldsymbol{\alpha}_{q_{3}}^{t} \boldsymbol{X}_{q_{3}}^{2} \boldsymbol{\alpha}_{q_{3}} \mathbf{1}_{q_{3}}^{t}\left(\boldsymbol{s}_{1_{q_{3}}}^{*}+\boldsymbol{s}_{2_{q_{3}}}^{*}\right)-\frac{\bar{Y}}{4} \boldsymbol{\alpha}_{q_{3}}^{t} \boldsymbol{X}_{q_{3}}^{2} \boldsymbol{\Sigma}_{q_{3}} \boldsymbol{\alpha}_{q_{3}} .
\end{aligned}
$$

The optimum values $\boldsymbol{\alpha}_{7_{q_{1}}}$ and $\boldsymbol{\alpha}_{7_{q_{3}}}$ are $\boldsymbol{\alpha}_{7_{q_{1}}}=\boldsymbol{\Delta}_{q_{1}}^{-1} \boldsymbol{\Phi}_{y x_{q_{3}}}+\boldsymbol{\Delta}_{q_{1}}^{-1} \boldsymbol{\Delta}_{q_{12}} \boldsymbol{\Delta}_{q_{2}}^{*-1} \boldsymbol{\Delta}_{q_{21}}^{-1}$. $\boldsymbol{\Delta}_{q_{1}}^{-1} \boldsymbol{\Phi}_{y x_{q_{1}}}-\boldsymbol{\Delta}_{q_{1}}^{-1} \boldsymbol{\Delta}_{q_{12}} \boldsymbol{\Delta}_{q_{2}}^{*-1} \boldsymbol{\Phi}_{y x_{q_{1}}}$ and $\boldsymbol{\alpha}_{7_{q_{3}}}=2 \bar{Y}^{-1} \boldsymbol{X}_{q_{2}}^{-1} \boldsymbol{\Delta}_{q_{2}}^{*-1}\left(\boldsymbol{\Phi}_{y x_{q_{2}}}-\boldsymbol{\Delta}_{q_{21}} \boldsymbol{\Delta}_{q_{1}}^{-1}\right.$. $\left.\boldsymbol{\Phi}_{y x_{q_{1}}}\right)$.

The mean square error of (4.16) can be obtained by substituting $a=d=$ $g=1, b=c=0$ in (4.5) and (4.6) and using (4.9) as

$$
\begin{aligned}
\operatorname{MSE}\left(t_{q_{r c r}}\right) \approx & \lambda_{2} S_{y}^{2}+\theta_{y}^{2}-\Phi_{y x_{q_{1}}}^{t} \Delta_{q_{1}}^{-1} \Phi_{y x_{q_{1}}} \\
& +\left(2 \Phi_{y x_{q_{2}}}^{t}-\Phi_{y x_{q_{1}}}^{t} B_{q_{12}}^{*}\right) \Delta_{q_{2}}^{*-1} B_{q_{21}}^{*} \Phi_{y x_{q_{1}}}^{t}-\Phi_{y x_{q_{2}}}^{t} \Delta_{q_{2}}^{*-1} \Phi_{y x_{q_{2}}}^{t}
\end{aligned}
$$

For $(a, d, b)=\{(1,1,1),(-1,1,-1),(-1,1,1)\}$ the regression-cum-exponentialproduct, difference-cum-exponential ratio and difference-cum-exponential-product estimator can be obtained as

$$
\begin{aligned}
& t_{q_{\text {rcep }}}=\left\{\bar{y}_{2}^{*}+\sum_{i=1}^{q_{1}} \alpha_{8_{i}}\left(\bar{x}_{1 i}^{*}-\bar{x}_{2 i}^{*}\right)\right\}\left\{\exp \sum_{i=q_{2}+1}^{q_{3}} \alpha_{8_{i}}\left(\frac{\left(\bar{x}_{2 i}^{*}-\bar{x}_{1 i}^{*}\right)}{\left(\bar{x}_{2 i}^{*}+\bar{x}_{1 i}^{*}\right)}\right)\right\}, \\
& t_{q_{\text {dcer }}}=\left\{\bar{y}_{2}^{*}+\sum_{i=1}^{q_{1}} \alpha_{9_{i}}\left(\bar{x}_{2 i}^{*}-\bar{x}_{1 i}^{*}\right)\right\}\left\{\exp \sum_{i=q_{2}+1}^{q_{3}} \alpha_{9_{i}}\left(\frac{\left(\bar{x}_{1 i}^{*}-\bar{x}_{2 i}^{*}\right)}{\left(\bar{x}_{2 i}^{*}+\bar{x}_{1 i}^{*}\right)}\right)\right\}
\end{aligned}
$$

and $t_{q_{\text {dcep }}}=\left\{\bar{y}_{2}^{*}+\sum_{i=1}^{q_{1}} \alpha_{10_{i}}\left(\bar{x}_{2 i}^{*}-\bar{x}_{1 i}^{*}\right)\right\}\left\{\exp \sum_{i=q_{2}+1}^{q_{3}} \alpha_{10_{i}}\left(\frac{\left(\bar{x}_{2 i}^{*}-\bar{x}_{1 i}^{*}\right)}{\left(\bar{x}_{2 i}^{*}+\bar{x}_{1 i}^{*}\right)}\right)\right\}$ respectively. The optimum values for $t_{m_{\text {rcp }}}$ are $\alpha_{8_{q_{1}}}=\alpha_{7_{q_{1}}}$ and $\alpha_{8_{q_{3}}}=-\alpha_{7_{q_{3}}}$, for $t_{m_{\text {dcer }}} \alpha_{9_{q_{1}}}=$ $-\alpha_{7_{1}}$ and $\alpha_{q_{q_{3} \times 1}}=\alpha_{7_{q_{3} \times 1}}$ and for $t_{m_{d c p}} \alpha_{10_{q_{1}}}=-\alpha_{7_{q_{1}}}$ and $\alpha_{10_{q_{3}}}=-\alpha_{7_{q_{3}}}$.

The bias and MSE of regression-cum-exponential-product, difference-cumexponential-ratio and difference-cum-exponential-product estimators are the same as for the estimators given in (4.19).

The expressions of bias and MSE for the above special cases can be obtained from (2.9) and (2.10) by using a suitable combination of $(a, b, c, d, g)$. The following table contains some existing estimators which are modified according to the situation discussed in this paper and are special cases of the proposed class. 
Table 1. Modified form of some existing estimators as special cases of the proposed class.

\begin{tabular}{cccc}
\hline Sr. \# & $\left(a, b, c, d, g, q_{1}, q_{2}, q_{3}\right)$ & Estimator & Reference \\
\hline 1 & $(0,0,0,1,0,0,0,0)$ & $\bar{y}_{2}^{*}$ & Hansen and Hurwitz (1946) \\
2 & $(0,1,1,0,0,0,1,0)$ & $\bar{y}_{2}^{*}\left(\frac{\bar{x}_{1}^{*}}{\bar{x}_{2}^{*}}\right)$ & Khare and Srivastava $(1993,1995)$ \\
3 & $(0,1,-1,0,0,0,1,0)$ & $\bar{y}_{2}^{*}\left(\frac{\bar{x}_{2}^{*}}{\bar{x}_{1}^{*}}\right)$ & \\
4 & $(1,0,0,1,0,1,0,0)$ & $\bar{y}_{2}^{*}+\alpha\left(\bar{x}_{1}^{*}+\bar{x}_{2}^{*}\right)$ & \\
5 & $(0,0,0,1,-1,0,0,1)$ & $\bar{y}_{2}^{*} \exp \left(\frac{\bar{x}_{1}^{*}-\bar{x}_{2}^{*}}{\bar{x}_{1}^{*}+\bar{x}_{2}^{*}}\right)$ & Singh et al. (2010) \\
6 & $(0,0,0,1,1,0,0,1)$ & $\bar{y}_{2}^{*} \exp \left(\frac{\bar{x}_{2}^{*}-\bar{x}_{1}^{*}}{\bar{x}_{2}^{*}+\bar{x}_{1}^{*}}\right)$ & \\
\hline
\end{tabular}

\subsection{Theoretical comparison for the proposed class}

In this section we compare our proposed class of estimators with Hansen and Hurwitz (1946). The comparison of the proposed class of estimators with Hansen and Hurwitz (1946) is:

$$
\operatorname{MSE}\left(\bar{y}_{2}^{*}\right)-\operatorname{MSE}\left(t_{q}\right)=\boldsymbol{\Phi}_{y x_{q}}^{t} \boldsymbol{T}_{q}^{-1} \boldsymbol{\Phi}_{y x_{q}}>0 .
$$

We can observe that our proposed class of estimators is more efficient than Hansen and Hurwitz (1946).

\section{Empirical comparison}

For empirical comparison, the data set of the Census Report of the Faisalabad District (1998) Pakistan has been used, which was also used by Ahmad et al. (2009b). For calculation of Bias and MSE of the estimators, it is assumed from 283 population units that $28 \%$ are non-respondents i.e. $N_{2}=80$ and remaining $N_{1}=203$ are respondents. The details of the study and auxiliary variables are given in Table A1. Each variable is taken from Rural Locality. The necessary results required for the calculation of bias and MSE are given in Tables A2, A3 and A4. The values of bias and percent relative efficiency of the proposed and existing estimators for different values of $n_{1}, n_{2}, k_{1}$ and $k_{2}$ are given in Tables A5 and $\mathrm{A} 6$.

For different combinations of $a, b, c, d$ and $g$ fourteen members of the proposed class have been considered for empirical comparison. As in the literature, the only case discussed was where non response was considered in the second phase. Therefore, for comparison purpose, estimators of the literature are modified according to the situation where non response is considered in both phases and empirical results are given in Section 5.

From Table A5, the MSE of six estimators (ratio, product, regression, difference, exponential, exponential ratio and exponential product) is the same and similarly, for eight remaining estimators is the same. The group of eight estimators is more efficient because the Percent Relative Efficiency (PRE) of 
Table A1. Description of variables (Each variable is taken from Rural Locality).

\begin{tabular}{ll}
\hline \multicolumn{1}{c}{ Description of variables } \\
\hline$Y_{2}$ & Population of currently married \\
$X_{1}$ & Population of both sexes \\
$X_{2}$ & Population of primary but below matric \\
$X_{3}$ & Population of matric and above \\
$X_{4}$ & Population of 18 years old and above \\
$X_{5}$ & Population of women 15-49 years old \\
\hline
\end{tabular}

Table A2. Average of all the Variables w.r.t. $N$ (283).

\begin{tabular}{ccccccc}
\hline Variables & $Y_{2}$ & $X_{1}$ & $X_{2}$ & $X_{3}$ & $X_{4}$ & $X_{5}$ \\
\hline Average & 1511.261 & 10931.44 & 1969.286 & 754.3604 & 6173.163 & 2457.682 \\
\hline
\end{tabular}

Table A3. Variance-covariance matrix w.r.t. $N$.

\begin{tabular}{ccccccc}
\hline & $Y_{2}$ & $X_{1}$ & $X_{2}$ & $X_{3}$ & $X_{4}$ & $X_{5}$ \\
\hline$Y_{2}$ & 621542.7328 & 3963614.8 & 792130.418 & 303104.0047 & 2481864.876 & 823818.0161 \\
$X_{1}$ & 3963614.842 & 29441849 & 5836183.44 & 2230311.546 & 17507723.7 & 6588651.038 \\
$X_{2}$ & 792130.4178 & 5836183.4 & 2815406.61 & 624113.7475 & 3788276.829 & 1390088.258 \\
$X_{3}$ & 303104.0047 & 2230311.5 & 624113.748 & 276331.9476 & 1795351.395 & 533963.3136 \\
$X_{4}$ & 2481864.876 & 17507724 & 3788276.83 & 1795351.395 & 39560446.26 & 4060606.428 \\
$X_{5}$ & 823818.0161 & 6588651 & 1390088.26 & 533963.3136 & 4060606.428 & 2196836.707 \\
\hline
\end{tabular}

Table A4. Variance-covariance matrix w.r.t. $N_{2}$.

\begin{tabular}{ccccccc}
\hline & $Y_{2}$ & $X_{1}$ & $X_{2}$ & $X_{3}$ & $X_{4}$ & $X_{5}$ \\
\hline$Y_{2}$ & 915368.5722 & 5470158.3 & 1207503.21 & 429915.8 & 3939985.41 & 1236879.163 \\
$X_{1}$ & 5470158.306 & 43396500 & 8701020.14 & 3059560.347 & 27495437.31 & 8863944.5 \\
$X_{2}$ & 1207503.209 & 8701020.1 & 2605859.46 & 788441.1218 & 6822705.087 & 1983612.909 \\
$X_{3}$ & 429915.8 & 3059560.3 & 788441.122 & 382683.9487 & 3553687.451 & 716956.6554 \\
$X_{4}$ & 3939985.41 & 27495437 & 6822705.09 & 3553687.451 & 120333017.3 & 6217267.278 \\
$X_{5}$ & 1236879.163 & 8863944.5 & 1983612.91 & 716956.6554 & 6217267.278 & 2000402.069 \\
\hline
\end{tabular}

this group is greater than the group consisting of six estimators. To check the performance within these two groups, there is a need to observe the bias of these from Table A6. This group of eight estimators having the same MSE can be further divided into two groups on the basis of biases, each consisting of four estimators. The first group of four estimators (Regression-cumexponentialratio, Regression-cum-exponential-product, difference-cum-exponential-ratio and difference-cumexponential-product) is preferred because it has less bias as compared to the second group of estimators (Regression-cum-ratio, Regression-cumproduct, difference-cum-ratio and difference-cum-product). 
Table A5. Results of the percent relative efficiency w.r.t. $\bar{y}_{2}^{*}$ of existing and proposed estimators.

\begin{tabular}{|c|c|c|c|c|c|c|c|}
\hline$k_{1}=k_{2}$ & $n_{1}$ & $n_{2}$ & $T_{R 1 d}$ & $T_{l r 1 d}$ & $t_{R(-1) d}^{(1)}$ & $\begin{array}{l}t_{l r_{1}}^{m}, t_{d_{1}}^{m}, t_{r_{1}}^{m} \\
t_{e p_{1}}^{m}, t_{e r_{1}}^{m}, t_{p_{1}}^{m}\end{array}$ & $\begin{array}{c}t_{r c r_{1}}^{m}, t_{r c p_{1}}^{m}, t_{d c r_{1}}^{m}, t_{d c p_{1}}^{m}, \\
t_{r c e r_{1}}^{m}, t_{r c e p_{1}}^{m}, t_{d c e r_{1}}^{m}, t_{d c e p_{1}}^{m}\end{array}$ \\
\hline 2 & 130 & 90 & 107.68 & 112.10 & 126.54 & 158.86 & 160.34 \\
\hline 2 & 130 & 100 & 105.85 & 108.99 & 119.41 & 138.68 & 139.53 \\
\hline 2 & 130 & 110 & 103.96 & 105.93 & 112.63 & 122.95 & 123.39 \\
\hline 2 & 130 & 120 & 102.01 & 102.94 & 106.16 & 110.34 & 110.52 \\
\hline 2 & 140 & 90 & 109.03 & 114.32 & 132.18 & 178.35 & 180.58 \\
\hline 2 & 140 & 100 & 107.35 & 111.37 & 125.19 & 154.71 & 156.06 \\
\hline 2 & 140 & 110 & 105.61 & 108.46 & 118.50 & 136.41 & 137.19 \\
\hline 2 & 140 & 120 & 103.81 & 105.60 & 112.08 & 121.81 & 122.23 \\
\hline 2 & 150 & 90 & 110.22 & 116.32 & 137.48 & 200.55 & 203.78 \\
\hline 2 & 150 & 100 & 108.68 & 113.52 & 130.68 & 172.82 & 174.83 \\
\hline 2 & 150 & 110 & 107.08 & 110.75 & 124.12 & 151.51 & 152.75 \\
\hline 2 & 150 & 120 & 105.41 & 108.02 & 117.78 & 134.63 & 135.36 \\
\hline 2 & 160 & 90 & 111.29 & 118.12 & 142.49 & 226.07 & 230.65 \\
\hline 2 & 160 & 100 & 109.87 & 115.47 & 135.89 & 193.44 & 196.33 \\
\hline 2 & 160 & 110 & 108.39 & 112.84 & 129.48 & 168.59 & 170.43 \\
\hline 2 & 160 & 120 & 106.86 & 110.23 & 123.26 & 149.03 & 150.19 \\
\hline 3 & 130 & 90 & 107.41 & 110.49 & 124.41 & 150.01 & 151.14 \\
\hline 3 & 130 & 100 & 105.59 & 107.79 & 117.75 & 132.37 & 133.01 \\
\hline 3 & 130 & 110 & 103.75 & 105.15 & 111.48 & 118.98 & 119.32 \\
\hline 3 & 130 & 120 & 101.89 & 102.55 & 105.57 & 108.47 & 108.61 \\
\hline 3 & 140 & 90 & 108.70 & 112.38 & 129.50 & 166.66 & 168.33 \\
\hline 3 & 140 & 100 & 107.02 & 109.83 & 122.94 & 145.74 & 146.75 \\
\hline 3 & 140 & 110 & 105.31 & 107.31 & 116.74 & 130.04 & 130.62 \\
\hline 3 & 140 & 120 & 103.57 & 104.84 & 110.87 & 117.81 & 118.12 \\
\hline 3 & 150 & 90 & 109.85 & 114.07 & 134.25 & 185.67 & 188.07 \\
\hline 3 & 150 & 100 & 108.29 & 111.66 & 127.83 & 160.82 & 162.30 \\
\hline 3 & 150 & 110 & 106.69 & 109.27 & 121.72 & 142.38 & 143.29 \\
\hline 3 & 150 & 120 & 105.07 & 106.91 & 115.90 & 128.16 & 128.70 \\
\hline 3 & 160 & 90 & 110.87 & 115.60 & 138.70 & 207.59 & 210.98 \\
\hline 3 & 160 & 100 & 109.42 & 113.31 & 132.43 & 177.95 & 180.05 \\
\hline 3 & 160 & 110 & 107.93 & 111.04 & 126.44 & 156.26 & 157.58 \\
\hline 3 & 160 & 120 & 106.42 & 108.79 & 120.70 & 139.69 & 140.52 \\
\hline
\end{tabular}

Now we want to check the rate of increase/decrease in Bias and MSE of the different estimators by increasing the values of $n_{1}, n_{2}, k_{1}$ and $k_{2}$ under the assumption that the trend will be linear. For this purpose, two values are considered for each $n_{1}, n_{2}, k_{1}$ and $k_{2}$ and slopes are calculated for Bias and MSE of all estimators. The results are given in Table A7.

From Table A7, by comparing our suggested and existing estimators, we can observe that when we increase the value of $k_{1}$ and $k_{2}$, the value of PRE of estimators decreased as expected. The decrease in PRE of $T_{R 1 d}$ is more when 
Table A5. (continued).

\begin{tabular}{|c|c|c|c|c|c|c|c|}
\hline$k_{1}=k_{2}$ & $n_{1}$ & $n_{2}$ & $T_{R 1 d}$ & $T_{l r 1 d}$ & $t_{R(-1) d}^{(1)}$ & $\begin{array}{l}t_{r_{1}}^{m}, t_{p_{1}}^{m}, t_{l r_{1}}^{m} \\
t_{d_{1}}^{m}, t_{e p_{1}}^{m}, t_{e r_{1}}^{m}\end{array}$ & $\begin{array}{c}t_{r c r_{1}}^{m}, t_{r c p_{1}}^{m}, t_{d c r_{1}}^{m}, t_{d c p_{1}}^{m}, \\
t_{r c e r_{1}}^{m}, t_{r c e p_{1}}^{m}, t_{d c e r_{1}}^{m}, t_{d c e p_{1}}^{m}\end{array}$ \\
\hline 4 & 130 & 90 & 107.25 & 112.10 & 123.24 & 144.14 & 145.07 \\
\hline 4 & 130 & 100 & 105.45 & 108.99 & 116.83 & 128.38 & 128.92 \\
\hline 4 & 130 & 110 & 103.64 & 105.93 & 110.85 & 116.56 & 116.84 \\
\hline 4 & 130 & 120 & 101.82 & 102.94 & 105.25 & 107.36 & 107.48 \\
\hline 4 & 140 & 90 & 108.52 & 114.32 & 128.02 & 158.76 & 160.14 \\
\hline 4 & 140 & 100 & 106.84 & 111.37 & 121.71 & 140.03 & 140.85 \\
\hline 4 & 140 & 110 & 105.14 & 108.46 & 115.78 & 126.14 & 126.62 \\
\hline 4 & 140 & 120 & 103.44 & 105.60 & 110.21 & 115.43 & 115.69 \\
\hline 4 & 150 & 90 & 109.64 & 116.32 & 132.47 & 175.43 & 177.39 \\
\hline 4 & 150 & 100 & 108.07 & 113.52 & 126.28 & 153.10 & 154.30 \\
\hline 4 & 150 & 110 & 106.48 & 110.75 & 120.43 & 136.77 & 137.51 \\
\hline 4 & 150 & 120 & 104.88 & 108.02 & 114.91 & 124.31 & 124.76 \\
\hline 4 & 160 & 90 & 110.64 & 118.12 & 136.63 & 194.60 & 197.34 \\
\hline 4 & 160 & 100 & 109.17 & 115.47 & 130.57 & 167.89 & 169.57 \\
\hline 4 & 160 & 110 & 107.68 & 112.84 & 124.81 & 148.65 & 149.72 \\
\hline 4 & 160 & 120 & 106.18 & 110.23 & 119.35 & 134.14 & 134.82 \\
\hline 5 & 130 & 90 & 107.15 & 110.49 & 122.48 & 139.81 & 140.64 \\
\hline 5 & 130 & 100 & 105.36 & 107.79 & 116.25 & 125.53 & 126.00 \\
\hline 5 & 130 & 110 & 103.57 & 105.15 & 110.46 & 114.86 & 115.11 \\
\hline 5 & 130 & 120 & 101.78 & 102.55 & 105.05 & 106.60 & 106.70 \\
\hline 5 & 140 & 90 & 108.40 & 112.38 & 127.08 & 152.91 & 154.11 \\
\hline 5 & 140 & 100 & 106.72 & 109.83 & 120.93 & 135.92 & 136.65 \\
\hline 5 & 140 & 110 & 105.04 & 107.31 & 115.19 & 123.40 & 123.83 \\
\hline 5 & 140 & 120 & 103.36 & 104.84 & 109.80 & 113.79 & 114.02 \\
\hline 5 & 150 & 90 & 109.51 & 114.07 & 131.34 & 167.76 & 169.45 \\
\hline 5 & 150 & 100 & 107.93 & 111.66 & 125.30 & 147.54 & 148.58 \\
\hline 5 & 150 & 110 & 106.35 & 109.27 & 119.63 & 132.84 & 133.48 \\
\hline 5 & 150 & 120 & 104.76 & 106.91 & 114.29 & 121.67 & 122.06 \\
\hline 5 & 160 & 90 & 110.49 & 115.60 & 135.32 & 184.76 & 187.09 \\
\hline 5 & 160 & 100 & 109.01 & 113.31 & 129.40 & 160.60 & 162.04 \\
\hline 5 & 160 & 110 & 107.52 & 111.04 & 123.80 & 143.32 & 144.23 \\
\hline 5 & 160 & 120 & 106.03 & 108.79 & 118.52 & 130.34 & 130.92 \\
\hline
\end{tabular}

compared to the proposed estimators and is less for the group of eight estimators. Increasing the value of $k_{1}$ and $k_{2}$, bias increases for all estimators as expected. The bias of $t_{R(-1) d}^{(1)}$ increases the most and the bias of the exponential product and exponential ratio increase the least.

Increases in the value of $n_{1}$ increases the PRE of all the estimators considered in the comparison. The increase is high in suggested estimators particularly in the group of eight estimators. In the case of bias, almost all of the suggested 
Table A6. Results of bias of existing and proposed estimators.

\begin{tabular}{|c|c|c|c|c|c|c|c|c|c|}
\hline$k_{1}=k_{2}$ & $n_{1}$ & $n_{2}$ & $T_{R 1 d}$ & $T_{l r 1 d}$ & $t_{R(-1) d}^{(1)}$ & $t_{r_{1}}^{m}, t_{p_{1}}^{m}$ & $t_{e p_{1}}^{m}, t_{e r_{1}}^{m}$ & $\begin{array}{l}t_{r c r_{1}}^{m}, t_{r c p_{1}}^{m}, \\
t_{d c r_{1}}^{m}, t_{d c p_{1}}^{m}\end{array}$ & $\begin{array}{l}t_{r c e r_{1}}^{m}, t_{r c e p_{1}}^{m}, \\
t_{d c e r_{1}}^{m}, t_{d c e p_{1}}^{m}\end{array}$ \\
\hline 2 & 130 & 90 & 1.56 & 0.00027 & 5.75 & 0.04 & -1.68 & 0.0662 & 0.063 \\
\hline 2 & 130 & 100 & 1.05 & 0.00019 & 3.88 & 0.02 & -1.13 & 0.0456 & 0.043 \\
\hline 2 & 130 & 110 & 0.64 & 0.00012 & 2.35 & 0.01 & -0.69 & 0.0287 & 0.026 \\
\hline 2 & 130 & 120 & 0.29 & 0.00006 & 1.08 & 0.01 & -0.31 & 0.0146 & 0.012 \\
\hline 2 & 140 & 90 & 1.81 & 0.00032 & 6.68 & 0.04 & -1.95 & 0.0761 & 0.074 \\
\hline 2 & 140 & 100 & 1.31 & 0.00023 & 4.81 & 0.03 & -1.40 & 0.0555 & 0.053 \\
\hline 2 & 140 & 110 & 0.89 & 0.00016 & 3.28 & 0.02 & -0.96 & 0.0386 & 0.036 \\
\hline 2 & 140 & 120 & 0.54 & 0.00011 & 2.00 & 0.01 & -0.58 & 0.0246 & 0.022 \\
\hline 2 & 150 & 90 & 2.03 & 0.00035 & 7.48 & 0.05 & -2.18 & 0.0847 & 0.082 \\
\hline 2 & 150 & 100 & 1.52 & 0.00027 & 5.61 & 0.03 & -1.64 & 0.0641 & 0.062 \\
\hline 2 & 150 & 110 & 1.11 & 0.00020 & 4.08 & 0.03 & -1.19 & 0.0472 & 0.045 \\
\hline 2 & 150 & 120 & 0.76 & 0.00014 & 2.80 & 0.02 & -0.82 & 0.0332 & 0.031 \\
\hline 2 & 160 & 90 & 2.22 & 0.00038 & 8.18 & 0.05 & -2.39 & 0.0923 & 0.090 \\
\hline 2 & 160 & 100 & 1.71 & 0.00030 & 6.31 & 0.04 & -1.84 & 0.0717 & 0.070 \\
\hline 2 & 160 & 110 & 1.30 & 0.00023 & 4.78 & 0.03 & -1.39 & 0.0548 & 0.053 \\
\hline 2 & 160 & 120 & 0.95 & 0.00017 & 3.50 & 0.02 & -1.02 & 0.0407 & 0.039 \\
\hline 3 & 130 & 90 & 1.99 & 0.00054 & 7.21 & -0.02 & -2.04 & 0.0791 & 0.076 \\
\hline 3 & 130 & 100 & 1.34 & 0.00037 & 4.87 & -0.02 & -1.38 & 0.0544 & 0.051 \\
\hline 3 & 130 & 110 & 0.81 & 0.00023 & 2.95 & -0.01 & -0.84 & 0.0342 & 0.031 \\
\hline 3 & 130 & 120 & 0.37 & 0.00011 & 1.35 & 0.00 & -0.38 & 0.0174 & 0.014 \\
\hline 3 & 140 & 90 & 2.31 & 0.00062 & 8.37 & -0.03 & -2.37 & 0.0910 & 0.088 \\
\hline 3 & 140 & 100 & 1.66 & 0.00045 & 6.03 & -0.02 & -1.71 & 0.066 & 0.063 \\
\hline 3 & 140 & 110 & 1.13 & 0.00031 & 4.11 & -0.01 & -1.16 & 0.046 & 0.043 \\
\hline 3 & 140 & 120 & 0.69 & 0.00020 & 2.51 & -0.01 & -0.71 & 0.029 & 0.026 \\
\hline 3 & 150 & 90 & 2.59 & 0.00069 & 9.37 & -0.03 & -2.65 & 0.101 & 0.099 \\
\hline 3 & 150 & 100 & 1.94 & 0.00052 & 7.03 & -0.02 & -1.99 & 0.077 & 0.074 \\
\hline 3 & 150 & 110 & 1.41 & 0.00038 & 5.11 & -0.02 & -1.45 & 0.056 & 0.054 \\
\hline 3 & 150 & 120 & 0.97 & 0.00027 & 3.52 & -0.01 & -1.00 & 0.040 & 0.037 \\
\hline 3 & 160 & 90 & 2.83 & 0.00076 & 10.25 & -0.03 & -2.90 & 0.110 & 0.108 \\
\hline 3 & 160 & 100 & 2.18 & 0.00059 & 7.91 & -0.03 & -2.24 & 0.086 & 0.083 \\
\hline 3 & 160 & 110 & 1.65 & 0.00045 & 5.99 & -0.02 & -1.70 & 0.066 & 0.063 \\
\hline 3 & 160 & 120 & 1.21 & 0.00033 & 4.39 & -0.01 & -1.24 & 0.049 & 0.046 \\
\hline
\end{tabular}

estimators are stable except $t_{R(-1) d}^{(1)}$. Increases in $n_{2}$ decreases the PRE for all estimators unexpectedly. The increase is high in the suggested estimators, especially in the group of eight. In the case of bias all are stable except $t_{R(-1) d}^{(1)}$. Furthermore, stability is high in the suggested estimators. 
Table A6. (continued).

\begin{tabular}{|c|c|c|c|c|c|c|c|c|c|}
\hline$k_{1}=k_{2}$ & $n_{1}$ & $n_{2}$ & $T_{R 1 d}$ & $T_{l r 1 d}$ & $t_{R(-1) d}^{(1)}$ & $t_{r_{1}}^{m}, t_{p_{1}}^{m}$ & $t_{e p_{1}}^{m}, t_{e r_{1}}^{m}$ & $\begin{array}{l}t_{r c r_{1}}^{m}, t_{r c p_{1}}^{m}, \\
t_{d c r_{1}}^{m}, t_{d c p_{1}}^{m}\end{array}$ & $\begin{array}{l}t_{r c e r_{1}}^{m}, t_{r c e p_{1}}^{m}, \\
t_{d c e r_{1}}^{m}, t_{d c e p_{1}}^{m}\end{array}$ \\
\hline 4 & 130 & 90 & 2.42 & 0.00080 & 8.67 & -0.09 & -2.38 & 0.091 & 0.088 \\
\hline 4 & 130 & 100 & 1.63 & 0.00054 & 5.85 & -0.06 & -1.60 & 0.063 & 0.059 \\
\hline 4 & 130 & 110 & 0.99 & 0.00033 & 3.55 & -0.04 & -0.97 & 0.039 & 0.036 \\
\hline 4 & 130 & 120 & 0.45 & 0.00016 & 1.63 & -0.02 & -0.45 & 0.020 & 0.016 \\
\hline 4 & 140 & 90 & 2.81 & 0.00092 & 10.06 & -0.10 & -2.76 & 0.105 & 0.102 \\
\hline 4 & 140 & 100 & 2.02 & 0.00067 & 7.25 & -0.07 & -1.99 & 0.077 & 0.073 \\
\hline 4 & 140 & 110 & 1.38 & 0.00046 & 4.94 & -0.05 & -1.35 & 0.053 & 0.050 \\
\hline 4 & 140 & 120 & 0.84 & 0.00029 & 3.02 & -0.03 & -0.83 & 0.034 & 0.031 \\
\hline 4 & 150 & 90 & 3.14 & 0.00103 & 11.27 & -0.11 & -3.09 & 0.117 & 0.114 \\
\hline 4 & 150 & 100 & 2.36 & 0.00078 & 8.45 & -0.08 & -2.32 & 0.088 & 0.086 \\
\hline 4 & 150 & 110 & 1.71 & 0.00057 & 6.15 & -0.06 & -1.69 & 0.065 & 0.062 \\
\hline 4 & 150 & 120 & 1.18 & 0.00040 & 4.23 & -0.04 & -1.16 & 0.046 & 0.043 \\
\hline 4 & 160 & 90 & 3.44 & 0.00113 & 12.33 & -0.12 & -3.38 & 0.127 & 0.125 \\
\hline 4 & 160 & 100 & 2.65 & 0.00087 & 9.51 & -0.09 & -2.61 & 0.099 & 0.096 \\
\hline 4 & 160 & 110 & 2.01 & 0.00066 & 7.20 & -0.07 & -1.98 & 0.076 & 0.073 \\
\hline 4 & 160 & 120 & 1.47 & 0.00049 & 5.28 & -0.05 & -1.45 & 0.056 & 0.053 \\
\hline 5 & 130 & 90 & 2.84 & 0.00106 & 10.13 & -0.15 & -2.69 & 0.103 & 0.099 \\
\hline 5 & 130 & 100 & 1.92 & 0.00072 & 6.84 & -0.10 & -1.81 & 0.071 & 0.067 \\
\hline 5 & 130 & 110 & 1.16 & 0.00044 & 4.14 & -0.06 & -1.10 & 0.045 & 0.041 \\
\hline 5 & 130 & 120 & 0.53 & 0.00021 & 1.90 & -0.03 & -0.50 & 0.023 & 0.019 \\
\hline 5 & 140 & 90 & 3.30 & 0.00122 & 11.76 & -0.17 & -3.12 & 0.119 & 0.115 \\
\hline 5 & 140 & 100 & 2.38 & 0.00089 & 8.46 & -0.13 & -2.25 & 0.087 & 0.083 \\
\hline 5 & 140 & 110 & 1.62 & 0.00061 & 5.77 & -0.09 & -1.53 & 0.060 & 0.057 \\
\hline 5 & 140 & 120 & 0.99 & 0.00038 & 3.53 & -0.05 & -0.94 & 0.038 & 0.035 \\
\hline 5 & 150 & 90 & 3.70 & 0.00137 & 13.17 & -0.20 & -3.49 & 0.133 & 0.129 \\
\hline 5 & 150 & 100 & 2.77 & 0.00103 & 9.88 & -0.15 & -2.62 & 0.100 & 0.097 \\
\hline 5 & 150 & 110 & 2.02 & 0.00075 & 7.18 & -0.11 & -1.91 & 0.074 & 0.071 \\
\hline 5 & 150 & 120 & 1.39 & 0.00052 & 4.94 & -0.07 & -1.31 & 0.052 & 0.048 \\
\hline 5 & 160 & 90 & 4.04 & 0.00150 & 14.40 & -0.21 & -3.82 & 0.145 & 0.141 \\
\hline 5 & 160 & 100 & 3.12 & 0.00116 & 11.11 & -0.16 & -2.95 & 0.112 & 0.109 \\
\hline 5 & 160 & 110 & 2.36 & 0.00088 & 8.42 & -0.12 & -2.23 & 0.086 & 0.083 \\
\hline 5 & 160 & 120 & 1.73 & 0.00065 & 6.17 & -0.09 & -1.64 & 0.064 & 0.061 \\
\hline
\end{tabular}

\section{Conclusions}

We can conclude from the above discussion regarding bias, relative efficiency and slopes that four classes of estimators (Regression-cum-exponentialratio, Regression-cum-exponential-product, difference-cum-exponentialratio and difference-cum-exponential-product) have less Bias and MSE than all suggested and existing estimators, and are also less sensitive to increasing the values of $n_{1}$, $n_{2}, k_{1}$ and $k_{2}$. Hence this group is preferred over all other considered estimators. 
Table A7. Slopes of Proposed and Existing Estimators

\begin{tabular}{llccc}
\hline \multicolumn{1}{c}{ Estimators } & $k_{1}=k_{2}$ & $n_{1}$ & \multicolumn{1}{c}{$n_{2}$} \\
\hline \multirow{6}{*}{ PREs } & 4.97 & 0.12 & -0.15 \\
& $T_{R 1 d}$ & 4.64 & 0.20 & -0.25 \\
& $T_{l r 1 d}$ & 4.80 & 0.43 & -0.54 \\
& $t_{R(-1) d}^{(1)}$ & & & \\
& $t_{r_{1}}^{m}, t_{p_{1}}^{m}, t_{l r_{1}}^{m}$, & 4.02 & 1.02 & -1.28 \\
& $t_{d_{1}}^{m}, t_{e p_{1}}^{m}, t_{e r_{1}}^{m}$ & & & \\
& $t_{r c r_{1}}^{m}, t_{r c p_{1}}^{m}, t_{d c r_{1}}^{m}, t_{d c p_{1}}^{m}$, & 3.99 & 1.05 & -1.31 \\
& $t_{r c e r_{1}}^{m}, t_{r c e p_{1}}^{m}, t_{d c e r_{1}}^{m}, t_{d c e p_{1}}^{m}$ & & & \\
\hline \multirow{5}{*}{ Bias } & $T_{R 1 d}$ & 5.07 & 0.03 & -0.06 \\
& $T_{l r 1 d}$ & 5.00 & 0.00 & 0.00 \\
& $t_{R(-1) d}^{(1)}$ & 5.24 & 0.11 & -0.21 \\
& $t_{r_{1}}^{m}, t_{p_{1}}^{m}$ & 4.99 & 0.00 & 0.00 \\
& $t_{e p_{1}}^{m}, t_{e r_{1}}^{m}$ & 4.95 & -0.03 & 0.06 \\
& $t_{r c r_{1}}^{m}, t_{r c p_{1}}^{m}, t_{d c r_{1}}^{m}, t_{d c p_{1}}^{m}$ & 5.00 & 0.00 & 0.00 \\
& $t_{r c e r_{1}}^{m}, t_{r c e p_{1}}^{m}, t_{d c e r_{1}}^{m}, t_{d c e p_{1}}^{m}$ & 5.00 & 0.00 & 0.00 \\
\hline
\end{tabular}

It is also found that on increase in first phase sample size improves the performance of estimators rather than increases in the size of the second phase sample. Increase in the re-contacted sample increases the performance of estimators.

\section{Acknowledgements}

The authors would like to thank the Editor and the two anonymous referees for carefully reading the paper and their suggestions.

\section{REFERENCES}

Ahmad, Z. and Hanif, M. (2010). Generalized multi-phase multivariate regression estimators for partial information case using multi-auxiliary variables, World Applied Sciences Journal, 10(3), 370-379.

Ahmad, Z., Hanif, M. and Ahmad, M. (2009a). Generalized multivariate ratio estimators for multi-phase sampling using multi-auxiliary variables, Pakistan Journal of Statistics, 25(4), $615-629$.

Ahmad, Z., Hanif, M. and Ahmad, M. (2009b). Generalized regression-cum-ratio estimators for two-phase sampling using multi-auxiliary variables, Pakistan Journal of Statistics, 25(2), $1-14$.

Ahmad, Z., Hanif, M. and Ahmad, M. (2010). Generalized multi-phase multivariate ratio estimators for partial information case using multi-auxiliary variables, Communications of the Korean Statistical Society, 17(5), 625-637.

Hansen, M. H. and Hurwitz, W. N. (1946). The problem of non-response in sample surveys, American Statistical Association, 41(236), 517-529.

Khare, B. B. and Sinha, B. B. (2009). On class of estimators for population mean using multiauxiliary characters in the presence of non response, Statistics in Transition, 10(1), 3-14.

Khare, B. B. and Sinha, R. R. (2011). Estimation of population mean using multi-auxiliary characters with subsampling the non-respondents, Statistics in Transition-New Series, 12(1), 45-56.

Khare, B. B. and Srivastava, S. (1993). Estimation of population mean using auxiliary character in presence of non-response, Natl. Acad. Sci. Lett. India, 16(3), 111-114. 
Khare, B. B. and Srivastava, S. (1995). Study of conventional and alternative two phase sampling ratio, product and regression estimators in the presence of non-response, Proc. Nat. Acad. Sci. (India), 65(A), 195-203.

Mohanty, S. (1967). Combination of regression and ratio estimate, J. Ind. Statist. Assoc., 5, 16-19.

Samiuddin, M. and Hanif, M. (2007). Estimation of population means in single and two-phase sampling with or without additional information, Pakistan. Journal of Statistics, 23(2), 99-118.

Singh, H. P. and Kumar, S. (2008). Estimation of mean in presence of non-response using two phase sampling scheme, Statistical Papers, DOI:10.1007/s00362-008-0140-5.

Singh, H. P., Kumar, S. and Kozak, M. (2010). Improved estimation of finite population mean using sub-sampling to deal with non-response in two phase sampling scheme, Commun. Statist. Theor. Meth., 39, 792-802.

Singh, R., Chauhan, P. and and Sawan, N. (2010). Ratio-product type exponential estimator for estimating finite population mean using information on auxiliary attribute, Unpublished manuscript.

Tabasum, R. and Khan, I. A. (2004). Double sampling for ratio estimation with non-response, J. Ind. Soc. Agril. Statist., 58(3), 300-306.

Tripathi, T. P. and Khare, B. B. (1997). Estimation of mean vector in the presence of nonresponse, Commun. Statist. Theor. Meth., 26(9), 2255-2269.

Wu, C. and Luan, Y. (2003). Optimal calibration estimators under two-phase sampling, Journal of Official Statistics, 19(2), 119-131. 